\title{
Trigonometric Polynomial Interpolation of Images
}

\author{
Thibaud Briand ${ }^{1,2}$ \\ ${ }^{1}$ Université Paris-Est, LIGM (UMR CNRS 8049), ENPC, F-77455 Marne-la-Vallée, France \\ ${ }^{2}$ Université Paris-Saclay, CMLA (UMR CNRS 8536), France \\ (briand.thibaud@gmail.com)
}

Communicated by Pascal Monasse and Jean-Michel Morel

Demo edited by Thibaud Briand

\begin{abstract}
For 1D and 2D signals, the Shannon-Whittaker interpolation with periodic extension can be formulated as a trigonometric polynomial interpolation (TPI). In this work, we describe and discuss the theory of TPI of images and some of its applications. First, the trigonometric polynomial interpolators of an image are characterized and it is shown that there is an ambiguity as soon as one size of the image is even. Three classical choices of interpolator for real-valued images are presented and cases where they coincide are pointed out. Then, TPI is applied to the geometric transformation of images, to up-sampling and to down-sampling. General results are expressed for any choice of interpolator but more details are given for the three proposed ones. It is proven that the well-known DFT-based computations have to be slightly adapted.
\end{abstract}

\section{Source Code}

The ANSI C99 implementation of the code that we provide is the one which has been peer reviewed and accepted by IPOL. The source code, the code documentation, and the online demo are accessible at the IPOL web page of this article ${ }^{1}$. Compilation and usage instructions are included in the README.txt file of the archive.

Keywords: interpolation; trigonometric polynomial; trigonometric polynomial interpolation; discrete Fourier transform (DFT); Non-equispaced fast Fourier transform (NFFT)

\section{Introduction}

Interpolation consists in constructing new data points within the range of a discrete set of known data points. It is closely related to the concept of approximation [7], fitting [9] and extrapolation. In signal processing it is commonly expressed as the problem of recovering the underlying continuous signal from which the known data points are sampled. Under the assumption that the signal belongs to a given class of functions, the common principle of all interpolation schemes is to determine the parameters of the continuous signal representation. In the following we focus on interpolation of uniformly sampled data on a regular grid, and in particular on image interpolation. The most common interpolation methods are presented in [23, 11].

\footnotetext{
${ }^{1}$ https://doi.org/10.5201/ipol.2019.273
} 
Shannon-Whittaker interpolation. A fundamental example of interpolation formula is given by Shannon-Whittaker's sampling theory [19, 24]. Let $d \in \mathbb{N}$ (typically 1 or 2 ).

Definition 1 (Fourier transform). The continuous Fourier transform applied to $L^{1}\left(\mathbb{R}^{d}\right)$ and its inverse, denoted $\mathcal{F}$ and $\mathcal{F}^{-1}$, are defined by

$$
\begin{gathered}
\forall u \in L^{1}\left(\mathbb{R}^{d}\right), \quad \forall \mathbf{y} \in \mathbb{R}^{d}, \quad \hat{u}(\mathbf{y})=\mathcal{F}(u)(\mathbf{y})=\int_{\mathbb{R}^{d}} u(\mathbf{x}) e^{-i \mathbf{x} \cdot \mathbf{y}} d \mathbf{x}, \\
\text { and } \quad \forall \mathbf{x} \in \mathbb{R}^{d}, \quad \mathcal{F}^{-1}(u)(\mathbf{x})=\frac{1}{(2 \pi)^{d}} \int_{\mathbb{R}^{d}} u(\mathbf{y}) e^{i \mathbf{x} \cdot \mathbf{y}} d \mathbf{y} .
\end{gathered}
$$

The Fourier transform extended to tempered distributions [18, 22] is still denoted $\mathcal{F}$.

Definition 2 (Band-limited distribution). A tempered distribution u is said to be band-limited if the support of its Fourier transform is bounded. It is said to be Nyquist band-limited if the support is contained in the Nyquist domain $[-\pi, \pi]^{d}$.

Theorem 1 (Shannon-Whittaker sampling theorem $[24,19])$. Let $u \in L^{1}\left(\mathbb{R}^{d}, \mathbb{C}\right)$ be a Nyquist bandlimited function. Then, $u$ is continuous and uniquely determined by its values at integer locations $\{u(\mathbf{k})\}_{\mathbf{k} \in \mathbb{Z}^{d}}$ since for $\mathbf{x} \in \mathbb{R}^{d}$,

$$
u(\mathbf{x})=\sum_{\mathbf{k} \in \mathbb{Z}^{d}} f(\mathbf{k}) \operatorname{sinc}(\mathbf{x}-\mathbf{k}),
$$

where the cardinal sine function sinc is the continuous function defined by

$$
\forall \mathbf{x}=\left(x_{1}, \ldots, x_{d}\right) \in\left(\mathbb{R}^{*}\right)^{d}, \quad \operatorname{sinc}(\mathbf{x})=\prod_{i=1}^{d} \frac{\sin \left(\pi x_{i}\right)}{\pi x_{i}} .
$$

Theorem 1, namely the Shannon-Whittaker sampling theorem, provides an equivalence between a band-limited function and its equidistant samples taken at a frequency that is superior or equal to the Nyquist rate. According to the Shannon-Whittaker interpolation formula (3), a band-limited signal can be written as the convolution between a Dirac comb weighted by its samples and the cardinal sine (or sinc) function. However, this result cannot be used directly because, among others, it requires an infinite number of samples [20]. The finite signal first needs to be arbitrarily extended. Among all the possible extensions a classical solution is the periodic extension. For 1D and 2D signals, the Shannon-Whittaker interpolation with periodic extension can be formulated as a trigonometric polynomial interpolation (TPI), namely the Discrete Shannon interpolation [1].

More generally, TPI is theoretically interesting since the band-limited periodic distributions are exactly the trigonometric polynomials. But the main advantage is practical. It is well known that the discrete Fourier transform (DFT) of a signal, which can be computed efficiently using the fast Fourier transform (FFT) algorithm [8], is deeply linked to TPI (or sampling). Efficient image processing algorithms that rely on DFT-based computations can be used, for instance, to perform linear filtering, up-sampling and down-sampling or to shift signals. More generally, any geometric transformation can be applied efficiently to images using the non-equispaced Fast Fourier transform (NFFT) algorithm [15], which is based on oversampled FFTs.

As soon as one of the dimensions is even, there is, however, an ambiguity in the definition of trigonometric polynomial interpolators (whose degree corresponds to the size of the image). The various interpolators differ from each other at the Nyquist frequency. Three particular interpolators are commonly used for real-valued images. The most common one is obtained directly from the DFT coefficients so that it is compatible with DFT-based computations but it may be complex-valued. Its real part is also a trigonometric polynomial interpolator and is usually used implicitly by taking 
the real part afterwards. The third interpolator is also real-valued and is given by the discrete Shannon interpolation. The choice of the interpolator depends on the context of application and may influence the results of algorithms relying on TPI (e.g. see [5]). Also, the compatibility with DFT-based computations has to be proven.

Contributions. This work is based on [3, Chapter 3]. We present the TPI of images and some of its applications. The trigonometric polynomial interpolators are characterized in terms of the DFT of the image. Three classical choices of interpolator for real-valued images are presented and cases where they coincide are pointed out. The theory is applied to the geometric transformations of images, and to up-sampling and down-sampling. General results are expressed for any choice of interpolator but more details are given for the three proposed interpolators. This study proves that the well-known DFT-based computations have to be slightly adapted.

The remainder of this paper is organized as follows: Section 2 presents the theory of TPI of images. This theory is applied to the geometric transformation of images in Section 3, and to up-sampling and down-sampling in Section 4.

Remark. In the following only grayscale images are considered. The results are easily extended to color (or multi-channel) images by handling channels independently.

\section{Trigonometric Polynomial Interpolation of Images}

In this section we present the theory of TPI for images. First, useful definitions and notations are introduced in Section 2.1. Then, the trigonometric polynomial interpolators of an image are characterized in Section 2.2 and it is shown that there is an ambiguity as soon as one of the dimensions is even. In Section 2.3 three classical choices of interpolator for real-valued images are proposed and cases where they coincide are pointed out.

\subsection{Definitions and Notations}

In the following $M$ and $N$ denote two positive integers. First, discrete domains are defined as in

Definition 3 (Discrete domains). The discrete spatial domain $\Omega_{M, N}$ is defined by

$$
\Omega_{M, N}=\{0, \ldots, M-1\} \times\{0, \ldots, N-1\} .
$$

The discrete Fourier domain $\hat{\Omega}_{M, N}$, associated to $\Omega_{M, N}$, is defined by $\hat{\Omega}_{M, N}=\hat{\Omega}_{M} \times \hat{\Omega}_{N}$ where for a positive integer $L$

$$
\hat{\Omega}_{L}= \begin{cases}\left\{-\frac{L-1}{2}, \ldots, \frac{L-1}{2}\right\} & \text { if } L \text { is odd, } \\ \left\{-\frac{L}{2}, \ldots, \frac{L}{2}-1\right\} & \text { if } L \text { is even. }\end{cases}
$$

The boundary $\Gamma_{M, N}$ of $\hat{\Omega}_{M, N}$ is defined by $\Gamma_{M, N}=\left(\Gamma_{M} \times \hat{\Omega}_{N}\right) \cup\left(\hat{\Omega}_{M} \times \Gamma_{N}\right)$ where for a positive integer $L$

$$
\Gamma_{L}= \begin{cases}\emptyset & \text { if } L \text { is odd, } \\ \left\{-\frac{L}{2}\right\} & \text { if } L \text { is even. }\end{cases}
$$

The symmetrized discrete Fourier domain $\hat{\Omega}_{M, N}^{s}$, associated to $\Omega_{M, N}$, is defined by $\hat{\Omega}_{M, N}^{s}=\hat{\Omega}_{M}^{s} \times$ $\hat{\Omega}_{N}^{s}$ where for a positive integer $L$

$$
\hat{\Omega}_{L}^{s}= \begin{cases}\hat{\Omega}_{L} & \text { if } L \text { is odd, } \\ \hat{\Omega}_{L} \cup\left\{\frac{L}{2}\right\} & \text { if } L \text { is even. }\end{cases}
$$


The boundary $\Gamma_{M, N}^{s}$ of $\hat{\Omega}_{M, N}^{s}$ is defined by $\Gamma_{M, N}^{s}=\hat{\Omega}_{M, N}^{s} \backslash\left(\hat{\Omega}_{M, N} \backslash \Gamma_{M, N}\right)$.

As an example, the discrete domains $\hat{\Omega}_{M, N}, \hat{\Omega}_{M, N}^{s}, \Gamma_{M, N}$ and $\Gamma_{M, N}^{s}$ for $M=N=4$ are displayed in Figure 1. Note that assuming that $M$ and $N$ are odd numbers, $\hat{\Omega}_{M, N}=\hat{\Omega}_{M, N}^{s}$ and $\Gamma_{M, N}=\Gamma_{M, N}^{s}=\emptyset$.

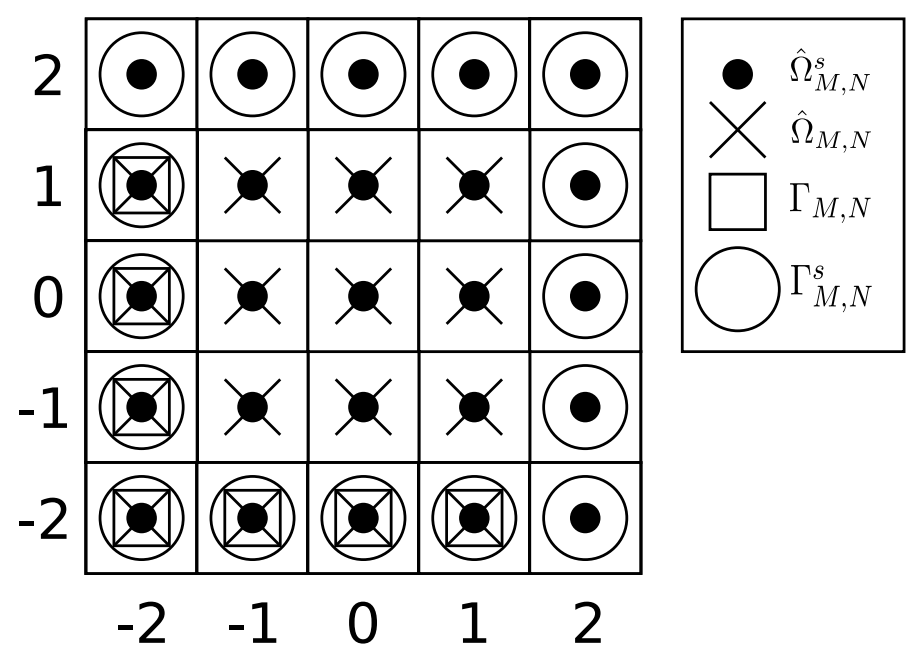

Figure 1: Discrete domains $\hat{\Omega}_{M, N}, \hat{\Omega}_{M, N}^{s}, \Gamma_{M, N}$ and $\Gamma_{M, N}^{s}$ for $M=N=4$.

Definition 4 (Image). An image (or digital image) $\underline{u}$ of size $M \times N$ is defined as a two-dimensional finite matrix of complex numbers $\left(\underline{u}_{k, l}\right)_{(k, l) \in \Omega_{M, N}}$. The image is said to be real-valued when every number is real.

In the following $\underline{u}$ denotes an image of size $M \times N$. Unless otherwise specified, $\underline{u}$ is not assumed to be real-valued. Trigonometric polynomial functions of the plane are defined as follows.

Definition 5 (Trigonometric polynomial). A function $P: \mathbb{R}^{2} \rightarrow \mathbb{C}$ is said to be a trigonometric polynomial of degree $\leq M \times N$ if there exists $c \in \mathbb{C}^{\hat{\Omega}_{M, N}^{s}}$, called coefficients of $P$, s.t.

$$
\forall(x, y) \in \mathbb{R}^{2}, \quad P(x, y)=\sum_{(m, n) \in \hat{\Omega}_{M, N}^{s}} c_{m, n} e^{2 i \pi\left(x \frac{m}{M}+y \frac{n}{N}\right)} .
$$

The set of trigonometric polynomials of degree $\leq M \times N$, denoted $\mathcal{P}_{M, N}$, is a subset of the set of $(M, N)$-periodic functions. Denote $\mathcal{R}$ the real part operator and let $P \in \mathcal{P}_{M, N}$ then $\mathcal{R}(P) \in \mathcal{P}_{M, N}$.

Note that another possible convention [16, p. 88] for trigonometric polynomials of degree $\leq M \times N$ is to consider $(2 \pi, 2 \pi)$-periodic functions of the form

$$
Q(x, y)=\sum_{(m, n) \in \hat{\Omega}_{2 M+1,2 N+1}} c_{m, n} e^{i(m x+n y)}=\sum_{m=-M}^{M} \sum_{n=-N}^{N} c_{m, n} e^{i(m x+n y)} .
$$

This convention is directly linked to ours (see Definition 5) since $(x, y) \in \mathbb{R}^{2} \mapsto Q\left(\frac{2 \pi}{M} x, \frac{2 \pi}{N} y\right)$ is a trigonometric polynomial of degree $\leq(2 M+1) \times(2 N+1)$. Note that the corresponding degree necessarily involves odd numbers. Our convention has the advantage of being compatible with the size of images and with the Discrete Fourier transform (DFT), which is defined as in 
Definition 6 (Discrete Fourier transform). The discrete Fourier transform (DFT) of $\underline{u} \in \mathbb{C}^{\Omega_{M, N}}$ is denoted $\mathcal{F}_{M, N}(\underline{u}) \in \mathbb{C}^{\hat{\Omega}_{M, N}}$ and is defined by

$$
\forall(m, n) \in \hat{\Omega}_{M, N}, \quad \mathcal{F}_{M, N}(\underline{u})_{m, n}=\frac{1}{M N} \sum_{(k, l) \in \Omega_{M, N}} \underline{u}_{k, l} e^{-2 \pi i\left(k \frac{m}{M}+l \frac{n}{N}\right)} .
$$

The inverse discrete Fourier transform (iDFT) of $\underline{v} \in \mathbb{C}^{\hat{\Omega}_{M, N}}$ is denoted $\mathcal{F}_{M, N}^{-1}(\underline{v}) \in \mathbb{C}^{\Omega_{M, N}}$ and is defined by

$$
\forall(k, l) \in \Omega_{M, N}, \quad \mathcal{F}_{M, N}^{-1}(\underline{v})_{k, l}=\sum_{(m, n) \in \hat{\Omega}_{M, N}} \underline{v}_{m, n} e^{2 \pi i\left(m \frac{k}{M}+n \frac{l}{N}\right)} .
$$

Note that another classical convention is obtained by moving the normalization factor $\frac{1}{M N}$ from the DFT to the iDFT. The resulting unnormalized DFT is used in the following to show the spectrum of images (for visualization purposes). The DFT and iDFT of size $M \times N$ can be computed efficiently in $\mathcal{O}(M N \log (M N))$ floating point operations thanks to the fast Fourier transform (FFT) algorithm [8].

\subsection{Trigonometric Polynomial Interpolators}

The trigonometric polynomial interpolators of an image $\underline{u}$ are defined and characterized as follows.

Definition 7 (Trigonometric polynomial interpolator). A trigonometric polynomial interpolator of $\underline{u}$ is a trigonometric polynomial $P \in \mathcal{P}_{M, N}$ verifying the interpolation condition

$$
\forall(k, l) \in \Omega_{M, N}, \quad P(k, l)=\underline{u}_{k, l} .
$$

By definition a trigonometric polynomial interpolator has a degree smaller than the size of the interpolated image. Note that if the degree is not controlled, the interpolation condition in (13) is always verified by infinitely many trigonometric polynomials.

Proposition 1. Let $P \in \mathcal{P}_{M, N} . P$ is a trigonometric polynomial interpolator of $\underline{u}$ if and only if its coefficients $c \in \mathbb{C}^{\hat{\Omega}_{M, N}^{s}}$ verify

$$
\begin{cases}c_{m, n}=\mathcal{F}_{M, N}(\underline{u})_{m, n} & \text { for }|m|<\frac{M}{2} \text { and }|n|<\frac{N}{2}, \\ c_{\frac{M}{2}, n}+c_{-\frac{M}{2}, n}=\mathcal{F}_{M, N}(\underline{u})_{-\frac{M}{2}, n} & \text { for }|n|<\frac{N}{2}, \\ c_{m, \frac{N}{2}}+c_{m,-\frac{N}{2}}=\mathcal{F}_{M, N}(\underline{u})_{m,-\frac{N}{2}} & \text { for }|m|<\frac{M}{2}, \\ c_{\frac{M}{2}, \frac{N}{2}}+c_{\frac{M}{2},-\frac{N}{2}}+c_{-\frac{M}{2}, \frac{N}{2}}+c_{-\frac{M}{2},-\frac{N}{2}}=\mathcal{F}_{M, N}(\underline{u})_{-\frac{M}{2},-\frac{N}{2}} . & \end{cases}
$$

Proof. Let $P \in \mathcal{P}_{M, N}$ be a trigonometric polynomial interpolator of $\underline{u}$. Let $(k, l) \in \Omega_{M, N}$. We have

$$
\underline{u}_{k, l}=P(k, l)=\sum_{(m, n) \in \hat{\Omega}_{M, N}^{s}} c_{m, n} e^{2 i \pi\left(k \frac{m}{M}+l \frac{n}{N}\right)} .
$$

Noting that for any $(L, j) \in \mathbb{N}^{*} \times \mathbb{N}$ we have $e^{2 i \pi j \frac{L}{2} \frac{1}{L}}=e^{2 i \pi j \frac{-L}{2} \frac{1}{L}}=(-1)^{j}$, we can write

$$
\underline{u}_{k, l}=\sum_{(m, n) \in \hat{\Omega}_{M, N}} d_{m, n} e^{2 i \pi\left(k \frac{m}{M}+l \frac{n}{N}\right)},
$$


where $d=\left(d_{m, n}\right) \in \mathbb{C}^{\hat{\Omega}_{M, N}}$ verify for $(m, n) \in \hat{\Omega}_{M, N}$,

$$
d_{m, n}= \begin{cases}c_{m, n} & \text { if }|m|<\frac{M}{2} \text { and }|n|<\frac{N}{2}, \\ c_{\frac{M}{2}, n}+c_{-\frac{M}{2}, n} & \text { if } m=-\frac{M}{2} \text { and }|n|<\frac{N}{2}, \\ c_{m, \frac{N}{2}}+c_{m,-\frac{N}{2}} & \text { if }|m|<\frac{M}{2} \text { and } n=-\frac{N}{2}, \\ c_{\frac{M}{2}, \frac{N}{2}}+c_{\frac{M}{2},-\frac{N}{2}}+c_{-\frac{M}{2}, \frac{N}{2}}+c_{-\frac{M}{2},-\frac{N}{2}} & \text { if } m=-\frac{M}{2} \text { and } n=-\frac{N}{2} .\end{cases}
$$

Finally by definition of the DFT and iDFT we have $\mathcal{F}_{M, N}^{-1}(d)=\underline{u}=\mathcal{F}_{M, N}^{-1}\left(\mathcal{F}_{M, N}(\underline{u})\right)$ and thus $d=\mathcal{F}_{M, N}(\underline{u})$.

Conversely, if $P \in \mathcal{P}_{M, N}$ is defined by the coefficients $c$ verifying (14) then it is an interpolator of $\underline{u}$.

The existence of trigonometric polynomial interpolators and their characterization is given by Proposition 1. They can easily be obtained from the DFT coefficients of the image so that the TPI is commonly called DFT interpolation.

Corollary 1. One of the two following cases occur.

- If $M$ and $N$ are odd, then there is a unique trigonometric polynomial interpolator noted $P_{\underline{u}}$ whose coefficients are $\mathcal{F}_{M, N}(\underline{u})$.

- If $M$ or $N$ is even, then there are infinitely many trigonometric polynomial interpolators. Their coefficients only differ from each other on the boundary $\Gamma_{M, N}^{s}$.

Proof. It is a direct consequence of Proposition 1.

When for instance $M$ is even, one can easily check that the interpolation property is kept when adding any multiple of the trigonometric polynomial of degree $M \times N$ defined by $(x, y) \in \mathbb{R}^{2} \mapsto$ $\sin (\pi x)$. As pointed out in [1] and [5], the lack of symmetry for even sizes introduces an ambiguity in the choice of the trigonometric polynomial interpolator.

This can also be seen with a dimensional approach. The space of trigonometric polynomials of degree $\leq M \times N$ has for dimension $\# \hat{\Omega}_{M, N}^{s}$, which is greater or equal to $M N=\# \Omega_{M, N}$. The linear map $P \in \mathcal{P}_{M, N} \mapsto(P(k, l))_{(k, l) \in \Omega_{M, N}}$ is one-to-one (and bijective) if and only if the kernel dimension \# $\left(\Gamma_{M, N}^{s} \backslash \Gamma_{M, N}\right)$ is 0 . Finally, it is one-to-one if and only if $M$ and $N$ are odd numbers. Actually $\# \hat{\Omega}_{M, N}^{s}$ is the number of coefficients $c_{m, n}$ used to represent trigonometric polynomials of degree $M \times N$ and is a product of odd numbers. If $M$ is even and $N$ is odd then the kernel dimension is $N$. If both $M$ and $N$ are even then the kernel dimension is $M+N+1$.

The dimensional approach also shows that $M \times N$ is the smallest degree (along both dimensions) ensuring the existence of a trigonometric polynomial verifying the interpolation condition (13).

\subsection{Trigonometric Polynomial Interpolators of a Real-valued Image}

Assume that $\underline{u}$ is real-valued. Three particular trigonometric polynomial interpolators of $\underline{u}$ are proposed and the cases where they coincide are pointed out.

Definition 8 (Trigonometric polynomial interpolator in complex convention). The trigonometric polynomial interpolator of $\underline{u}$ in complex convention is the trigonometric polynomial $P_{\underline{u}}^{(c)} \in \mathcal{P}_{M, N}$ defined by

$$
\forall(x, y) \in \mathbb{R}^{2}, \quad P_{\underline{u}}^{(c)}(x, y)=\sum_{(m, n) \in \hat{\Omega}_{M, N}} \mathcal{F}_{M, N}(\underline{u})_{m, n} e^{2 i \pi\left(x \frac{m}{M}+y \frac{n}{N}\right)} .
$$


The trigonometric polynomial interpolator in complex convention is the natural and simplest way to define a trigonometric polynomial interpolator since the coefficients are directly expressed in terms of the DFT of the image. Using Proposition 1 it is proven to be an interpolator (even for a complex-valued image) since it corresponds to the particular case where the coefficients are null in $\Gamma_{M, N}^{s} \backslash \Gamma_{M, N}$. However, because of the DFT asymmetry, it may be complex-valued. Therefore two other interpolators, which are guaranteed to be real-valued, are built.

Proposition 2. Assume $\underline{u}$ is real-valued. Then, $\mathcal{R}\left(P_{\underline{u}}^{(c)}\right) \in \mathcal{P}_{M, N}$ is a trigonometric polynomial interpolator of $\underline{u}$ whose coefficients $c \in \hat{\Omega}_{M, N}^{s}$ verify for $(m, n) \in \hat{\Omega}_{M, N}^{s}$,

$$
c_{m, n}= \begin{cases}\mathcal{F}_{M, N}(\underline{u})_{m, n} & \text { if }|m|<\frac{M}{2} \text { and }|n|<\frac{N}{2}, \\ \frac{1}{2} \mathcal{F}_{M, N}(\underline{u})_{-\frac{M}{2}, n} & \text { if }|m|=\frac{M}{2} \text { and }|n|<\frac{N}{2}, \\ \frac{1}{2} \mathcal{F}_{M, N}(\underline{u})_{m,-\frac{N}{2}} & \text { if }|m|<\frac{M}{2} \text { and }|n|=\frac{N}{2}, \\ \frac{1}{2} \mathcal{F}_{M, N}(\underline{u})_{-\frac{M}{2},-\frac{N}{2}} & \text { if }(m, n) \in\left\{ \pm\left(\frac{M}{2}, \frac{N}{2}\right)\right\}, \\ 0 & \text { if }(m, n) \in\left\{ \pm\left(-\frac{M}{2}, \frac{N}{2}\right)\right\} .\end{cases}
$$

Proof. This is a consequence of the Hermitian symmetry of the DFT of real-valued images. Indeed, extending the DFT by $(M, N)$-periodicity we easily get $\mathcal{F}_{M, N}(\underline{u})_{m, n}=\overline{\mathcal{F}_{M, N}(\underline{u})_{-m,-n}}$ for all $(m, n) \in$ $\mathbb{Z}^{2}$. Let $(x, y) \in \mathbb{R}^{2}$. By grouping the terms of the sum in Equation (18) we can deduce that the complex contribution of $P_{\underline{u}}^{(c)}$ comes from the terms with indices in $\Gamma_{M, N}$,

$$
\begin{aligned}
P_{\underline{u}}^{(c)}(x, y)= & \underbrace{\mathcal{F}_{M, N}(\underline{u})_{0,0}+\sum_{\begin{array}{c}
0 \leq m<\frac{M}{2}, \\
0 \leq n<\frac{N}{2} \\
(m, n) \neq(0,0)
\end{array}} 2 \mathcal{R}\left(\mathcal{F}_{M, N}(\underline{u})_{m, n} e^{2 i \pi\left(x \frac{m}{M}+y \frac{n}{N}\right)}\right)}_{\in \mathbb{R}} \\
& +\sum_{(m, n) \in \Gamma_{M, N}} \mathcal{F}_{M, N}(\underline{u})_{m, n} e^{2 i \pi\left(x \frac{m}{M}+y \frac{n}{N}\right)} .
\end{aligned}
$$

Case 1: Assume $M$ and $N$ are odd. Then $\Gamma_{M, N}=\emptyset$. This implies that $P_{\underline{u}}^{(c)}$ is real-valued and we have the result.

Case 2: Assume that $M$ is even and $N$ is odd. Then $\Gamma_{M, N}=\left\{-\frac{M}{2}\right\} \times\left\{-\frac{N-1}{2}, \ldots, \frac{N-1}{2}\right\}$. Using the relation $\mathcal{F}_{M, N}(\underline{u})_{-\frac{M}{2}, n}=\overline{\mathcal{F}_{M, N}(\underline{u})_{-\frac{M}{2},-n}}$ we have

$$
\sum_{(m, n) \in \Gamma_{M, N}} \mathcal{F}_{M, N}(\underline{u})_{m, n} e^{2 i \pi\left(x \frac{m}{M}+y \frac{n}{N}\right)}=e^{-i \pi x} \underbrace{\left(\mathcal{F}_{M, N}(\underline{u})_{-\frac{M}{2}, 0}+\sum_{n=1}^{\frac{N-1}{2}} 2 \mathcal{R}\left(\mathcal{F}_{M, N}(\underline{u})_{-\frac{M}{2}, n} e^{2 i \pi \frac{n y}{N}}\right)\right)}_{\in \mathbb{R}} .
$$

Finally as $\mathcal{R}\left(e^{-i \pi x}\right)=\frac{1}{2}\left(e^{i \pi x}+e^{-i \pi x}\right)=\frac{1}{2}\left(e^{2 i \pi \frac{M}{2} \frac{x}{M}}+e^{2 i \pi\left(-\frac{M}{2}\right) \frac{x}{M}}\right)$, we have

$$
\mathcal{R}\left(\sum_{(m, n) \in \Gamma_{M, N}} \mathcal{F}_{M, N}(\underline{u})_{m, n} e^{2 i \pi\left(x \frac{m}{M}+y \frac{n}{N}\right)}\right)=\sum_{(m, n) \in \Gamma_{M, N}^{s}} \frac{1}{2} \mathcal{F}_{M, N}(\underline{u})_{m, n} e^{2 i \pi\left(x \frac{m}{M}+y \frac{n}{N}\right)},
$$

and the result is obtained by identification. Similarly, we deal with the case $M$ odd and $N$ even by switching the coordinates. 
Case 3: Assume that $M$ and $M$ are even. Then we have the partition

$$
\Gamma_{M, N}=\left\{-\frac{M}{2}\right\} \times\left\{-\frac{N}{2}+1, \ldots, \frac{N}{2}-1\right\} \bigsqcup\left\{-\frac{M}{2}+1, \ldots, \frac{M}{2}-1\right\} \times\left\{-\frac{N}{2}\right\} \bigsqcup\left\{-\frac{M}{2}\right\} \times\left\{-\frac{N}{2}\right\},
$$

so that the sum $\sum_{(m, n) \in \Gamma_{M, N}} \mathcal{F}_{M, N}(\underline{u})_{m, n} e^{2 i \pi\left(x \frac{m}{M}+y \frac{n}{N}\right)}$ can be decomposed into three sums. The two first components can be handled as in case 2 (even though the sum indices are slightly different). The third component corresponds to the index $\left(-\frac{M}{2},-\frac{N}{2}\right)$ for which we have

$$
\begin{aligned}
\mathcal{R}\left(\mathcal{F}_{M, N}(\underline{u})_{-\frac{M}{2},-\frac{N}{2}} e^{-i \pi(x+y)}\right) & =\underbrace{\mathcal{F}_{M, N}(\underline{u})_{-\frac{M}{2},-\frac{N}{2}}}_{\in \mathbb{R}} \mathcal{R}\left(e^{-i \pi(x+y)}\right) \\
& =\frac{1}{2} \mathcal{F}_{M, N}(\underline{u})_{-\frac{M}{2},-\frac{N}{2}}\left(e^{i \pi(x+y)}+e^{-i \pi(x+y)}\right) .
\end{aligned}
$$

Finally the result is obtained by identification.

As stated in Proposition 2, $\mathcal{R}\left(P_{\underline{u}}^{(c)}\right)$ is a real-valued trigonometric polynomial interpolator of $\underline{u}$ which can be easily obtained from the complex convention. However, when $M$ and $N$ are even, its coefficients show an asymmetry in the highest frequencies. Therefore the following alternative may be preferred.

Definition 9 (Trigonometric polynomial interpolator in real convention). Assume $\underline{u}$ is real-valued. The trigonometric polynomial interpolator of $\underline{u}$ in real convention is defined as the trigonometric polynomial $P_{\underline{u}}^{(r)} \in \mathcal{P}_{M, N}$ whose coefficients $c \in \hat{\widehat{\Omega}}_{M, N}^{s}$ verify for $(m, n) \in \hat{\Omega}_{M, N}^{s}$,

$$
c_{m, n}= \begin{cases}\mathcal{F}_{M, N}(\underline{u})_{m, n} & \text { if }|m|<\frac{M}{2} \text { and }|n|<\frac{N}{2}, \\ \frac{1}{2} \mathcal{F}_{M, N}(\underline{u})_{-\frac{M}{2}, n} & \text { if }|m|=\frac{M}{2} \text { and }|n|<\frac{N}{2}, \\ \frac{1}{2} \mathcal{F}_{M, N}(\underline{u})_{m,-\frac{N}{2}} & \text { if }|m|<\frac{M}{2} \text { and }|n|=\frac{N}{2}, \\ \frac{1}{4} \mathcal{F}_{M, N}(\underline{u})_{-\frac{M}{2},-\frac{N}{2}} & \text { if }|m|=\frac{M}{2} \text { and }|n|=\frac{N}{2} .\end{cases}
$$

Proposition 3. Assume $\underline{u}$ is real-valued. Then $P_{\underline{u}}^{(r)}$ is a real-valued interpolating function of $\underline{u}$ since for $(x, y) \in \mathbb{R}^{2}$,

$$
P_{\underline{u}}^{(r)}(x, y)= \begin{cases}\mathcal{R}\left(P_{\underline{u}}^{(c)}\right)(x, y)+\mathcal{F}_{M, N}(\underline{u})_{-\frac{M}{2},-\frac{N}{2}} \sin (\pi x) \sin (\pi y) & \text { if } M \text { and } N \text { are even }, \\ \mathcal{R}\left(P_{\underline{u}}^{(c)}\right)(x, y) & \text { otherwise. }\end{cases}
$$

Proof. Noticing that $\mathcal{F}_{M, N}(\underline{u})_{-\frac{M}{2},-\frac{N}{2}} \in \mathbb{R}$ and using the relation

$$
\mathcal{R}\left(e^{-i \pi(x+y)}\right)+\sin (\pi x) \sin (\pi y)=\frac{1}{4}\left(e^{i \pi(x+y)}+e^{-i \pi(x+y)}+e^{i \pi(x-y)}+e^{i \pi(-x+y)}\right),
$$

it is directly obtained from the definition of $P_{\underline{u}}^{(r)}$ and Proposition 2 .

Proposition 3 states that $P_{\underline{u}}^{(r)}$ is another real-valued trigonometric polynomial interpolator and makes the link with $\mathcal{R}\left(P_{\underline{u}}^{(c)}\right)$. When $M$ and $N$ are even $P_{\underline{u}}^{(r)}$ is usually preferred to $\mathcal{R}\left(P_{\underline{u}}^{(c)}\right)$ because it has the same DFT coefficients at the four corners of $\hat{\Omega}_{M, N}^{s}$. In [1] the trigonometric polynomial interpolator in real convention is called discrete Shannon interpolator because it corresponds to the Shannon-Whittaker interpolator with periodic boundary extension.

The particular conditions under which the proposed trigonometric polynomial interpolators are equal are presented in Proposition 4. 
Proposition 4. Assume $\underline{u}$ is real-valued. The cases of equality of the three proposed trigonometric polynomial interpolators are:

1. $P_{\underline{u}}^{(c)}=\mathcal{R}\left(P_{\underline{u}}^{(c)}\right)=P_{\underline{u}}^{(r)}$ if and only if

$$
\left\{(m, n) \in \Gamma_{M, N} \mid \mathcal{F}_{M, N}(\underline{u})_{m, n} \neq 0\right\}=\emptyset
$$

In particular, it is the case when $M$ and $N$ are odd since there is a unique trigonometric polynomial interpolator and $\Gamma_{M, N}=\emptyset$.

2. Assume that $M$ or $N$ is odd. Then, $\mathcal{R}\left(P_{\underline{u}}^{(c)}\right)=P_{\underline{u}}^{(r)}$.

3. Assume that $M$ and $N$ are even. Then $\mathcal{R}\left(P_{\underline{u}}^{(c)}\right)=P_{\underline{u}}$ if and only if $\mathcal{F}_{M, N}(\underline{u})_{-\frac{M}{2},-\frac{N}{2}}=0$.

Proof. It is a direct consequence of Proposition 2 and Proposition 3.

\section{Application to Geometric Transformations of Images}

A continuous signal representation, thus interpolation, is required when one wishes to implement numerically an operator that is initially defined in the continuous domain. In particular, this representation is required when applying a geometric transformation to an image. Denote by $\sigma\left(\mathbb{R}^{2}\right)$ the set of bijective functions of $\mathbb{R}^{2}$ to itself. A function $\varphi \in \sigma\left(\mathbb{R}^{2}\right)$ is called a geometric transformation. Applying the geometric transformation $\varphi$ to the image $\underline{u}$ consists in resampling $\underline{u}$ at locations $\varphi^{-1}(k, l)$ for $(k, l) \in \Omega_{M, N}$. In general $\varphi^{-1}(k, l) \in \mathbb{R}^{2}$ does not belong to the integer grid and a continuous representation of $\underline{u}$ is required.

In this section we apply TPI to geometric transformations of images. Let $\underline{u}$ be an image of size $M \times N$. Let $P \in \mathcal{P}_{M, N}$ be a trigonometric polynomial interpolator of $\underline{u}$ with coefficients $c \in \hat{\Omega}_{M, N}^{s}$ and $\varphi \in \sigma\left(\mathbb{R}^{2}\right)$ be a geometric transformation. The transformation of $\underline{u}$ by $\varphi$ using $P$ is noted $\underline{u}_{P, \varphi}$ and is defined by

$$
\forall(k, l) \in \Omega_{M, N}, \quad\left(\underline{u}_{P, \varphi}\right)_{k, l}=P\left(\varphi^{-1}(k, l)\right) .
$$

First, the case of translations is considered in Section 3.1. It is shown that a translated image can be computed efficiently using DFT-based computations. In particular the results for the three classical trigonometric interpolators introduced in Section 2.3 are detailed. The invertibility of the translation operation is also studied. Then, an efficient algorithm for computing any transformation of an image is proposed in Section 3.2.

\subsection{Translation}

Let $(\alpha, \beta) \in \mathbb{R}^{2}$ be a shift parameter. The translation by $(\alpha, \beta)$ corresponds to the geometric transformation $\varphi:(x, y) \in \mathbb{R}^{2} \mapsto(x+\alpha, y+\beta)$. For simplicity we use slightly different notation for $\underline{u}_{P, \varphi}$ as proposed in

Definition 10. The translated image of $\underline{u}$ with shift $(\alpha, \beta)$ using the interpolator $P$ is noted $\underline{u}_{(P, \alpha, \beta)} \in$ $\mathbb{C}^{\Omega_{M, N}}$. It is defined by

$$
\forall(k, l) \in \Omega_{M, N}, \quad\left(\underline{u}_{(P, \alpha, \beta)}\right)_{k, l}=P(k-\alpha, l-\beta) .
$$


Proposition 5. The DFT coefficients of $\underline{u}_{(P, \alpha, \beta)}$ verify

$$
\begin{cases}\mathcal{F}_{M, N}\left(\underline{u}_{(P, \alpha, \beta)}\right)_{m, n}=c_{m, n} e^{-2 i \pi\left(\alpha \frac{m}{M}+\beta \frac{n}{N}\right)} & \text { for }|m|<\frac{M}{2} \text { and }|n|<\frac{N}{2}, \\ \mathcal{F}_{M, N}\left(\underline{u}_{(P, \alpha, \beta)}\right)_{-\frac{M}{2}, n}=\left(c_{-\frac{M}{2}, n} e^{i \pi \alpha}+c_{\frac{M}{2}, n} e^{-i \pi \alpha}\right) e^{-2 i \pi \beta \frac{n}{N}} & \text { for }|n|<\frac{N}{2}, \\ \mathcal{F}_{M, N}\left(\underline{u}_{(P, \alpha, \beta)}\right)_{m,-\frac{N}{2}}=\left(c_{m,-\frac{N}{2}} e^{i \pi \beta}+c_{m, \frac{N}{2}} e^{-i \pi \beta}\right) e^{-2 i \pi \alpha \frac{m}{M}} & \text { for }|m|<\frac{M}{2}, \\ \mathcal{F}_{M, N}\left(\underline{u}_{(P, \alpha, \beta)}\right)_{-\frac{M}{2},-\frac{N}{2}}=c_{-\frac{M}{2},-\frac{N}{2}} e^{i \pi(\alpha+\beta)}+c_{\frac{M}{2}, \frac{N}{2}} e^{-i \pi(\alpha+\beta)} & \\ \quad+c_{-\frac{M}{2}, \frac{N}{2}} e^{i \pi(\alpha-\beta)}+c_{\frac{M}{2},-\frac{N}{2}} e^{-i \pi(\alpha-\beta)} . & \end{cases}
$$

Proof. For $(k, l) \in \Omega_{M, N}$ we have

$$
\begin{aligned}
\left(\underline{u}_{(P, \alpha, \beta)}\right)_{k, l} & =P(k-\alpha, l-\beta) \\
& =\sum_{(m, n) \in \hat{\Omega}_{M, N}^{s}} c_{m, n} e^{2 i \pi\left(\frac{m(k-\alpha)}{M}+\frac{n(l-\beta)}{N}\right)} \\
& =\sum_{(m, n) \in \hat{\Omega}_{M, N}^{s}} c_{m, n} e^{-2 i \pi\left(\alpha \frac{m}{M}+\beta \frac{n}{N}\right)} e^{2 i \pi\left(\frac{m k}{M}+\frac{n l}{N}\right)} .
\end{aligned}
$$

The result is obtained using the same reasoning as in the proof of Proposition 1 except that the $c_{m, n}$ are multiplied by $e^{-2 i \pi\left(\alpha \frac{m}{M}+\beta \frac{n}{N}\right)}$.

As stated in Proposition 5, the DFT coefficients of the translated image $\underline{u}_{(P, \alpha, \beta)}$ can be easily computed from the trigonometric polynomial coefficients $c$ by a phase shift. Therefore the translation using TPI is commonly called the DFT translation. The coefficients in $\Gamma_{M, N}$ have a slightly different expression that depends on the choice of the interpolator. In particular, for the three classical trigonometric polynomial interpolators the DFT coefficients of the translated images are given by

Proposition 6. For all $(m, n) \in \hat{\Omega}_{M, N}$,

$$
\mathcal{F}_{M, N}\left(\underline{u}_{\left(P_{\underline{u}}^{(c)}, \alpha, \beta\right)}\right)_{m, n}=\mathcal{F}_{M, N}(\underline{u})_{m, n} e^{-2 i \pi\left(\alpha \frac{m}{M}+\beta \frac{n}{N}\right)} .
$$

If in addition $\underline{u}$ is real-valued, then

$$
\begin{cases}\mathcal{F}_{M, N}\left(\underline{u}_{\left(\mathcal{R}\left(P_{\underline{u}}^{(c)}\right), \alpha, \beta\right)}\right)_{m, n}=\mathcal{F}_{M, N}(\underline{u})_{m, n} e^{-2 i \pi\left(\alpha \frac{m}{M}+\beta \frac{n}{N}\right)} & \text { for }|m|<\frac{M}{2} \text { and }|n|<\frac{N}{2}, \\ \mathcal{F}_{M, N}\left(\underline{u}_{\left(\mathcal{R}\left(P_{\underline{u}}^{(c)}\right), \alpha, \beta\right)}\right)_{-\frac{M}{2}, n}=\mathcal{F}_{M, N}(\underline{u})_{-\frac{M}{2}, n} \cos (\pi \alpha) e^{-2 i \pi \beta \frac{n}{N}} & \text { for }|n|<\frac{N}{2}, \\ \mathcal{F}_{M, N}\left(\underline{u}_{\left(\mathcal{R}\left(P_{\underline{u}}^{(c)}\right), \alpha, \beta\right)}\right)_{m,-\frac{N}{2}}=\mathcal{F}_{M, N}(\underline{u})_{m,-\frac{N}{2}} \cos (\pi \beta) e^{-2 i \pi \alpha \frac{m}{M}} & \text { for }|m|<\frac{M}{2}, \\ \mathcal{F}_{M, N}\left(\underline{u}_{\left(\mathcal{R}\left(P_{\underline{u}}^{(c)}\right), \alpha, \beta\right)}\right)_{-\frac{M}{2},-\frac{N}{2}}=\mathcal{F}_{M, N}\left(\underline{u}_{-\frac{M}{2},-\frac{N}{2}} \cos (\pi(\alpha+\beta)),\right. & \end{cases}
$$

and

$$
\begin{cases}\mathcal{F}_{M, N}\left(\underline{u}_{\left(P_{\underline{u}}^{(r)}, \alpha, \beta\right)}\right)_{m, n}=\mathcal{F}_{M, N}(\underline{u})_{m, n} e^{-2 i \pi\left(\alpha \frac{m}{M}+\beta \frac{n}{N}\right)} & \text { for }|m|<\frac{M}{2} \text { and }|n|<\frac{N}{2}, \\ \mathcal{F}_{M, N}\left(\underline{u}_{\left(P_{\underline{u}}^{(r)}, \alpha, \beta\right)}\right)_{-\frac{M}{2}, n}=\mathcal{F}_{M, N}(\underline{u})_{-\frac{M}{2}, n} \cos (\pi \alpha) e^{-2 i \pi \beta \frac{n}{N}} & \text { for }|n|<\frac{N}{2}, \\ \mathcal{F}_{M, N}\left(\underline{u}_{\left(P_{\underline{u}}^{(r)}, \alpha, \beta\right)}\right)_{m,-\frac{N}{2}}=\mathcal{F}_{M, N}(\underline{u})_{m,-\frac{N}{2}} \cos (\pi \beta) e^{-2 i \pi \alpha \frac{m}{M}} & \text { for }|m|<\frac{M}{2}, \\ \mathcal{F}_{M, N}\left(\underline{u}_{\left(P_{\underline{u}}^{(r)}, \alpha, \beta\right)}\right)_{-\frac{M}{2},-\frac{N}{2}}= & \\ \mathcal{F}_{M, N}(\underline{u})_{-\frac{M}{2},-\frac{N}{2}} \frac{1}{2}(\cos (\pi(\alpha+\beta))+\cos (\pi(\alpha-\beta))) . & \end{cases}
$$

Proof. It is a direct consequence of Proposition 5. The coefficients of the trigonometric polynomial interpolators are given in Definition 8, Proposition 2 and Definition 9. 
Note that $\underline{u}_{\left(P_{\underline{u}}^{(c)}, \alpha, \beta\right)}$ may be a complex-valued image while $\underline{u}_{\left(\mathcal{R}\left(P_{\underline{\underline{x}}}^{(c)}, \alpha, \beta\right)\right.}$ and $\underline{u}_{\left(P_{\underline{u}}^{(r)}, \alpha, \beta\right)}$ are both real-valued images.

Using Proposition 6 it is possible to determine whether or not the DFT translation can be inverted by applying the DFT translation with opposite shift. It is the case for the trigonometric polynomial interpolator in complex convention as stated in

Proposition 7. Set $\underline{v}=\underline{u}_{\left(P_{\underline{u}}^{(c)}, \alpha, \beta\right)}$. Then,

$$
\underline{v}_{\left(P_{\underline{v}}^{(c)},-\alpha,-\beta\right)}=\underline{u} .
$$

Proof. It is obtained using (36) successively for $\underline{u}$ with $(\alpha, \beta)$, and then for $\underline{v}$ with $-(\alpha, \beta)$.

On the contrary for the two classical real-valued trigonometric polynomial interpolators it is not automatically the case. The DFT coefficients of the image obtained after the two opposite translations may differ from the original ones on the Fourier boundary $\Gamma_{M, N}$. More precisely, the DFT coefficients are given by

Proposition 8. Assume $\underline{u}$ is real-valued. Set $\underline{v}=\underline{u}_{\left(\mathcal{R}\left(P_{\underline{u}}^{(c)}\right), \alpha, \beta\right)}$. Then, $\underline{v}_{\left(\mathcal{R}\left(P_{\underline{v}}^{(c)}\right),-\alpha,-\beta\right)}$ verifies

$$
\begin{cases}\mathcal{F}_{M, N}\left(\underline{v}_{\left(\mathcal{R}\left(P_{\underline{v}}^{(c)}\right),-\alpha,-\beta\right)}\right)_{m, n}=\mathcal{F}_{M, N}(\underline{u})_{m, n} & \text { for }|m|<\frac{M}{2} \text { and }|n|<\frac{N}{2}, \\ \mathcal{F}_{M, N}\left(\underline{v}_{\left(\mathcal{R}\left(P_{\underline{v}}^{(c)}\right),-\alpha,-\beta\right)}\right)_{-\frac{M}{2}, n}=\cos (\pi \alpha)^{2} \mathcal{F}_{M, N}(\underline{u})_{-\frac{M}{2}, n} & \text { for }|n|<\frac{N}{2}, \\ \mathcal{F}_{M, N}\left(\underline{v}_{\left(\mathcal{R}\left(P_{\underline{v}}^{(c)}\right),-\alpha,-\beta\right)}\right)_{m,-\frac{N}{2}}=\cos (\pi \beta)^{2} \mathcal{F}_{M, N}(\underline{u})_{m,-\frac{N}{2}} & \text { for }|m|<\frac{M}{2}, \\ \mathcal{F}_{M, N}\left(\underline{v}_{\left(\mathcal{R}\left(P_{\underline{v}}^{(c)}\right),-\alpha,-\beta\right)}\right)_{-\frac{M}{2},-\frac{N}{2}}=\cos (\pi(\alpha+\beta))^{2} \mathcal{F}_{M, N}\left(\underline{u}_{-\frac{M}{2},-\frac{N}{2}} .\right. & \end{cases}
$$

Set $\underline{w}=\underline{u}_{\left(P_{\underline{u}}^{(r)}, \alpha, \beta\right)}$. Then, $\underline{w}_{\left(P_{\underline{w}}^{(r)},-\alpha,-\beta\right)}$ verifies

$$
\begin{cases}\mathcal{F}_{M, N}\left(\underline{w}_{\left(\left(P_{\underline{w}}^{(r)},-\alpha,-\beta\right)\right.}\right)_{m, n}=\mathcal{F}_{M, N}(\underline{u})_{m, n} & \text { for }|m|<\frac{M}{2} \text { and }|n|<\frac{N}{2}, \\ \mathcal{F}_{M, N}\left(\underline{w}_{\left(\left(P_{\underline{w}}^{(r)},-\alpha,-\beta\right)\right.}\right)_{-\frac{M}{2}, n}=\cos (\pi \alpha)^{2} \mathcal{F}_{M, N}(\underline{u})_{-\frac{M}{2}, n} & \text { for }|n|<\frac{N}{2}, \\ \mathcal{F}_{M, N}\left(\underline{w}_{\left(\left(P_{\underline{w}}^{(r)},-\alpha,-\beta\right)\right.}\right)_{m,-\frac{N}{2}}=\cos (\pi \beta)^{2} \mathcal{F}_{M, N}(\underline{u})_{m,-\frac{N}{2}} & \text { for }|m|<\frac{M}{2}, \\ \mathcal{F}_{M, N}\left(\underline{w}_{\left(\left(P_{\underline{w}}^{(r)},-\alpha,-\beta\right)\right.}\right)_{-\frac{M}{2},-\frac{N}{2}}= & \\ \quad \frac{1}{4}(\cos (\pi(\alpha+\beta))+\cos (\pi(\alpha-\beta)))^{2} \mathcal{F}_{M, N}(\underline{u})_{-\frac{M}{2},-\frac{N}{2}} . & \end{cases}
$$

Proof. It is a direct consequence of (37) and (38) applied successively to $(\alpha, \beta)$ and $-(\alpha, \beta)$.

The particular cases where a DFT translation is inverted by the opposite DFT translation can be summarized as in

Proposition 9. Assume $\underline{u}$ is real-valued. Let $P \in\left\{P_{\underline{u}}^{(c)}, \mathcal{R}\left(P_{\underline{u}}^{(c)}\right), P_{\underline{u}}^{(r)}\right\}$. Then, the following propositions are equivalent:

(1) The translation of $\underline{u}$ with shift $(\alpha, \beta)$ using the interpolator $P$ can be inverted by the opposite translation.

(2) For all $(m, n) \in \hat{\Omega}_{M, N}$,

$$
\left|\mathcal{F}_{M, N}\left(\underline{u}_{(P, \alpha, \beta)}\right)\right|_{m, n}=\left|\mathcal{F}_{M, N}(\underline{u})\right|_{m, n} .
$$

(3) For all $(m, n) \in \Gamma_{M, N}$,

$$
\left|\mathcal{F}_{M, N}\left(\underline{u}_{(P, \alpha, \beta)}\right)\right|_{m, n}=\left|\mathcal{F}_{M, N}(\underline{u})\right|_{m, n} .
$$


In particular, (1) holds as soon as:

1. $P=P_{\underline{u}}^{(c)}$.

2. $(\alpha, \beta) \in \mathbb{Z}^{2}$.

3. $P_{\underline{u}}^{(c)}=\mathcal{R}\left(P_{\underline{u}}^{(c)}\right)=P_{\underline{u}}^{(r)}$ i.e.

$$
\left\{(m, n) \in \Gamma_{M, N} \mid \mathcal{F}_{M, N}(\underline{u})_{m, n} \neq 0\right\}=\emptyset .
$$

In particular it is the case when $M$ and $N$ are odd.

4. $\beta \in \mathbb{Z}$ and

$$
\left\{n \in \Gamma_{N} \mid \mathcal{F}_{M, N}(\underline{u})_{-\frac{M}{2}, n} \neq 0\right\}=\emptyset .
$$

In particular it is the case when $N$ is odd.

5. $\alpha \in \mathbb{Z}$ and

$$
\left\{m \in \Gamma_{M} \mid \mathcal{F}_{M, N}(\underline{u})_{m,-\frac{N}{2}} \neq 0\right\}=\emptyset .
$$

In particular it is the case when $M$ is odd.

Proof. Set $\underline{v}=\underline{u}_{(P, \alpha, \beta)}$ and $\underline{w}=\underline{v}_{\left(P^{\prime},-\alpha,-\beta\right)}$ where $P^{\prime}$ is the corresponding trigonometric polynomial interpolator of $\underline{v}$. Using Proposition 6 we can write

$$
\mathcal{F}_{M, N}(\underline{v})=h(\alpha, \beta) \mathcal{F}_{M, N}(\underline{u}) .
$$

Set $h^{\prime}(\alpha, \beta)=|h(\alpha, \beta)|^{2}$. Using Proposition 8 we have

$$
\mathcal{F}_{M, N}(\underline{w})=h^{\prime}(\alpha, \beta) \mathcal{F}_{M, N}(\underline{u}) .
$$

Thus, (1) holds if and only if $\underline{w}=\underline{u}$ if and only if for all $(m, n) \in \Omega_{M, N}, h^{\prime}(\alpha, \beta)_{m, n}=1$ or $\mathcal{F}_{M, N}(\underline{u})=0$. As $h^{\prime}(\alpha, \beta)=|h(\alpha, \beta)|^{2},(1)$ holds if and only if for all $(m, n) \in \Omega_{M, N},|h(\alpha, \beta)|_{m, n}=1$ or $\mathcal{F}_{M, N}(\underline{u})=0$. Using (47) we obtain the equivalence of (1) and (2). For all $(m, n) \in \Omega_{M, N} \backslash \Gamma_{M, N}$, $h(\alpha, \beta)_{m, n}=e^{-2 i \pi\left(\alpha \frac{m}{M}+\beta \frac{n}{N}\right)}$ so that $|h(\alpha, \beta)|_{m, n}=1$. This shows that (2) and (3) are equivalent.

The verification of the particular cases is straightforward using Proposition 8.

How to deal with the non-invertibility of the DFT translation. The non-invertibility may be avoided by working with images with odd sizes, which is not always possible, or by killing the DFT coefficients on the boundary $\Gamma_{M, N}$, which modifies the image content. Alternatively it is possible to take into account the effect of the non-invertibility on the output result. For instance in [3, Chapter $6]$ we proposed a measure of the interpolation reversibility where high frequency components are discarded before the comparison.

\subsection{Efficient Image Transformation Algorithm}

The transformed image $\underline{u}_{P, \varphi}$ of $\underline{u}$ by $\varphi$ using $P$ is given by (30). The interpolated values correspond to the evaluation of the trigonometric polynomial $P$ at locations $\left\{\varphi^{-1}(k, l)\right\}_{(k, l) \in \Omega_{M, N}}$, which are a priori non-equispaced. As stated in Corollary 1, the coefficients $c$ of $P$ are expressed in terms of the DFT of $\underline{u}$. When $\varphi$ is a translation it was shown in Section 3.1 that $\underline{u}_{P, \varphi}$ can be computed by phase shift and an inverse DFT. Using the FFT algorithm [8] this can be obtained in $\mathcal{O}(M N \log (M N))$ floating point operations. The corresponding algorithm is presented in Algorithm 1.

As described below, in general the computation of the transformed image is more costly but can be approximated with an efficient algorithm. 


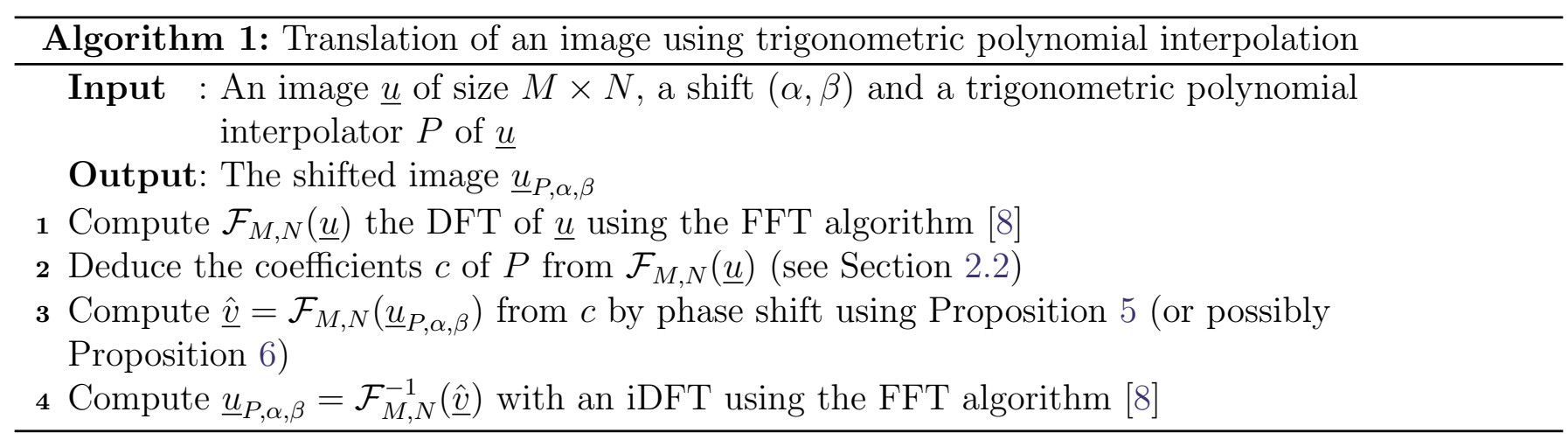

Trigonometric polynomial evaluation. Let us consider the general problem of trigonometric polynomial evaluation at arbitrary locations. Let $N_{0}$ be the number of output values and $\left\{\left(x_{j}, y_{j}\right)\right\}_{1 \leq j \leq N_{0}}$ be the locations. Then, $P$ can be evaluated at $\left(x_{j}, y_{j}\right)$ directly from the coefficients using the formula

$$
P\left(x_{j}, y_{j}\right)=\sum_{(m, n) \in \hat{\Omega}_{M, N}^{s}} c_{m, n} e^{2 i \pi\left(\frac{m x_{j}}{M}+\frac{n y_{j}}{N}\right)} .
$$

The total cost is of $\mathcal{O}\left(M N N_{0}\right)$ floating point operations so that this operation cannot be done in practice.

Non-equispaced discrete Fourier transform. Let us introduce the non-equispaced discrete Fourier transform (NDFT) algorithm. Let $M^{\prime}$ and $N^{\prime}$ be two even numbers and $\left(\hat{f}_{m, n}\right)_{(m, n) \in \hat{\Omega}_{M^{\prime}, N^{\prime}}}$. Let $\left\{\left(x_{j}^{\prime}, y_{j}^{\prime}\right)\right\}_{1 \leq j \leq N_{0}}$ be pixel positions in $\left[-\frac{1}{2}, \frac{1}{2}\right)^{2}$. The NDFT evaluates the sums

$$
f\left(x_{j}^{\prime}, y_{j}^{\prime}\right)=\sum_{(m, n) \in \hat{\Omega}_{M^{\prime}, N^{\prime}}} \hat{f}_{m, n} e^{-2 i \pi\left(x_{j}^{\prime} m+y_{j}^{\prime} n\right)} .
$$

The evaluation could be done using the straightforward matrix form and would require $\mathcal{O}\left(M^{\prime} N^{\prime} N_{0}\right)$ floating point operations.

It is clear that the expressions in (49) and (50) are closely related. Actually $P$ can be evaluated using the NDFT algorithm. The correspondences between positions are given by

$$
\left\{\begin{array}{l}
x_{j}^{\prime}=-\frac{x_{j}}{M}+\alpha_{j} \\
y_{j}^{\prime}=-\frac{y_{j}}{N}+\beta_{j}
\end{array}\right.
$$

where $\alpha_{j}$ and $\beta_{j}$ are integers insuring $\left(x_{j}^{\prime}, y_{j}^{\prime}\right) \in\left[-\frac{1}{2}, \frac{1}{2}\right)^{2}$. The even bandwidth $M^{\prime}$ and $N^{\prime}$ sizes are taken as

$$
M^{\prime}=\left\{\begin{array}{ll}
M+1 & \text { if } \mathrm{M} \text { is odd, } \\
M+2 & \text { if } \mathrm{M} \text { is even, }
\end{array} \quad \text { and } \quad N^{\prime}= \begin{cases}N+1 & \text { if } \mathrm{N} \text { is odd } \\
N+2 & \text { if } \mathrm{N} \text { is even }\end{cases}\right.
$$

The Fourier coefficients $\left(\hat{f}_{m, n}\right)_{(m, n) \in \hat{\Omega}_{M^{\prime}, N^{\prime}}}$ are obtained from $\left(c_{m, n}\right)_{(m, n) \in \hat{\Omega}_{M, N}^{s}}$ by zero-padding on the boundary $\Gamma_{M^{\prime}, N^{\prime}}$ i.e.

$$
\hat{f}_{m, n}= \begin{cases}c_{m, n} & (m, n) \in \hat{\Omega}_{M, N}^{s}, \\ 0 & (m, n) \in \Gamma_{M^{\prime}, N^{\prime}}\end{cases}
$$

The interest of considering the NDFT formulation is that it can be efficiently approximated by the non-equispaced fast Fourier transform [15] (NFFT) algorithm. The NFFT approximation is based on the usage of an oversampled FFT and a window function which is simultaneously localized in space 
and frequency. It only requires $\mathcal{O}\left(M^{\prime} N^{\prime} \log \left(M^{\prime} N^{\prime}\right)+|\log (\varepsilon)|^{2} N_{0}\right)$ operations where $\varepsilon$ denotes the desired relative output precision (in practice it is close to the machine precision). Details concerning the NFFT performances are provided in [13].

How to transform an image. Algorithm 2 details how the image $\underline{u}$ is transformed by $\varphi$ using the trigonometric polynomial interpolator $P$. First the locations $\left\{\varphi^{-1}(k, l)\right\}_{(k, l) \in \Omega_{M, N}}$ are computed and mapped into $\left[-\frac{1}{2}, \frac{1}{2}\right)^{2}$ using (51). Then, the DFT coefficients of $\underline{u}$ are computed (thanks to the FFT algorithm [8] in $\mathcal{O}(M N \log (M N))$ floating point operations) and linked to the coefficients of $P$. Finally the values $\left(\underline{u}_{P, \varphi}\right)_{k, l}=P\left(\varphi^{-1}(k, l)\right)$ are approximated on $\Omega_{M, N}$ using the NFFT algorithm using the correspondences provided in (52) and (53). The total number of floating point operations is in $\mathcal{O}\left(M N \log (M N)+|\log (\varepsilon)|^{2} M N\right)$.

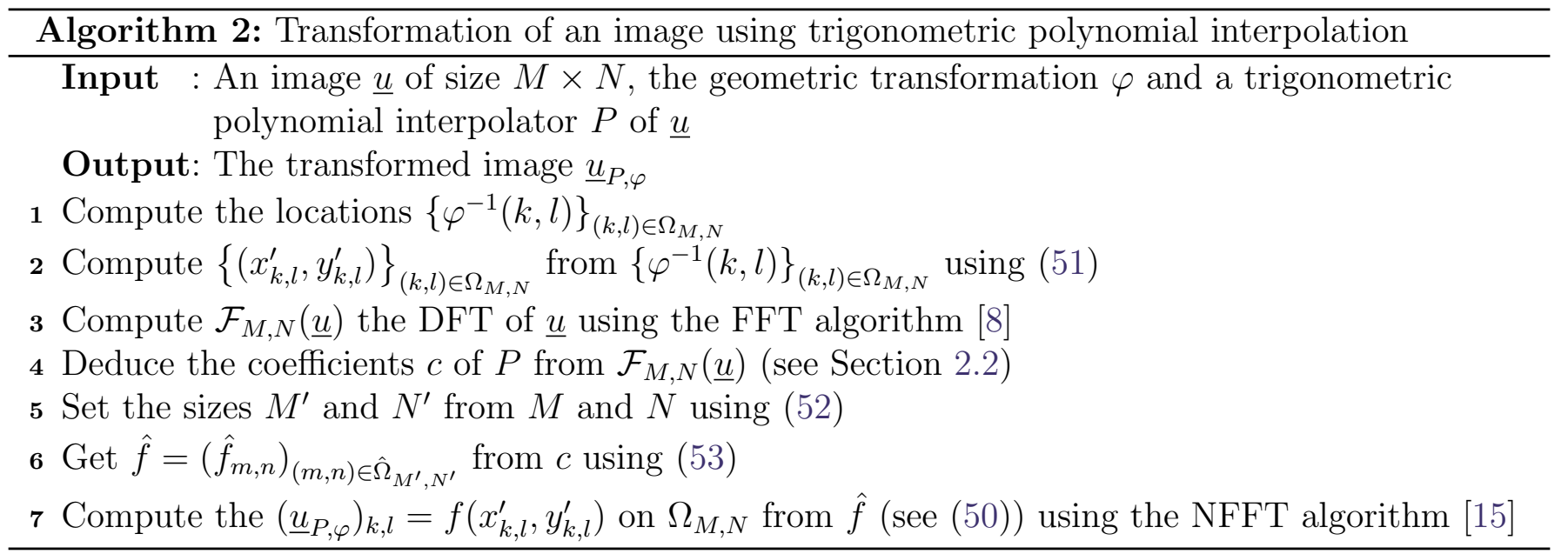

Particular cases. Assume that the trigonometric polynomial interpolator $P$ is one of the three classical interpolators i.e. $P \in\left\{P_{\underline{u}}^{(c)}, \mathcal{R}\left(P_{\underline{u}}^{(c)}\right), P_{\underline{u}}^{(r)}\right\}$. When $P=P_{\underline{u}}^{(c)}$ unnecessary computations are saved during the NFFT by keeping even bandwidths. In practice $M^{\prime}$ and $N^{\prime}$, which were set in Line 5 of Algorithm 2, are replaced by

$$
M^{\prime}=\left\{\begin{array}{ll}
M+1 & \text { if } \mathrm{M} \text { is odd, } \\
M & \text { if } \mathrm{M} \text { is even, }
\end{array} \text { and } \quad N^{\prime}= \begin{cases}N+1 & \text { if } \mathrm{N} \text { is odd } \\
N & \text { if } \mathrm{N} \text { is even }\end{cases}\right.
$$

The results for $P \in\left\{\mathcal{R}\left(P_{\underline{u}}^{(c)}\right), P_{\underline{u}}^{(r)}\right\}$ are obtained directly from the case $P=P_{\underline{u}}^{(c)}$ by taking the real part and by possibly using (27).

\section{Up-sampling and Down-sampling}

Up-sampling and down-sampling are common operations in image processing. For instance in multiscale approaches the down-sampling factor corresponds to the pyramid scale (e.g. see [6]). These operations cannot be interpreted as geometric transformations as described in Section 3 since the image sizes change. However they involve a spatial scaling and are closely related to zooming. That is why it is common to (improperly) refer to up-sampling as a zoom-in and to down-sampling as a zoom-out.

In this section we present the up-sampling and down-sampling using TPI. It is shown that DFT-based computations can be performed and the link between the two operations is established. 


\section{$4.1 \quad$ Up-sampling}

Here up-sampling refers to the process of increasing the sampling rate of a signal/image. As no additional information is provided, up-sampling can be seen as resampling on a finer grid using interpolation. Let $P \in \mathcal{P}_{M, N}$ be a trigonometric polynomial interpolator of $\underline{u}$ with coefficients $c \in \hat{\Omega}_{M, N}$. Let $\lambda_{x}$ and $\lambda_{y}$ be two numbers greater than 1 such that

$$
\left\{\begin{array}{l}
M^{\prime} \doteq \lambda_{x} M \in \mathbb{N} \\
N^{\prime} \doteq \lambda_{y} N \in \mathbb{N}
\end{array}\right.
$$

Actually the up-sampling factors $\lambda_{x}=\frac{M^{\prime}}{M}$ and $\lambda_{y}=\frac{N^{\prime}}{N}$ are rational numbers and any up-sampled size $M^{\prime} \geq M$ and $N^{\prime} \geq N$ can be obtained. The up-sampling of $\underline{u}$ by the up-sampling factor $\lambda=\left(\lambda_{x}, \lambda_{y}\right)$ using $P$ is defined as in

Definition 11 (Up-sampling of an image). Let $\lambda=\left(\lambda_{x}, \lambda_{y}\right) \in\left[1,+\infty\left[^{2}\right.\right.$ verifying (55). The upsampled image of $\underline{u}$ by factor $\lambda$ using the interpolator $P \in \mathcal{P}_{M, N}$ is noted $\underline{u}_{(P, \lambda)} \in \mathbb{C}^{\Omega_{M^{\prime}, N^{\prime}}}$ and is defined by

$$
\forall(k, l) \in \Omega_{M^{\prime}, N^{\prime}}, \quad\left(\underline{u}_{(P, \lambda)}\right)_{k, l}=P\left(\frac{k}{\lambda_{x}}, \frac{l}{\lambda_{y}}\right) .
$$

The DFT coefficients of the up-sampled image $\underline{u}_{(P, \lambda)}$ can be easily computed from the trigonometric polynomial coefficients by padding with zeros as stated in

Proposition 10. Let $\lambda=\left(\lambda_{x}, \lambda_{y}\right) \in\left[1,+\infty\left[{ }^{2}\right.\right.$ verifying $(55)$. Then, the DFT coefficients $\mathcal{F}_{M^{\prime}, N^{\prime}}\left(\underline{u}_{(P, \lambda)}\right)$ are obtained as follows:

1. If $\lambda_{x}=\lambda_{y}=1$, then $\underline{u}_{(P, \lambda)}=\underline{u}$ and $\mathcal{F}_{M^{\prime}, N^{\prime}}\left(\underline{u}_{(P, \lambda)}\right)=\mathcal{F}_{M, N}(\underline{u})$.

2. If $\lambda_{x}=1$ and $\lambda_{y}>1$, then $M^{\prime}=M$ and for $(m, n) \in \hat{\Omega}_{M^{\prime}, N^{\prime}}$,

$$
\mathcal{F}_{M^{\prime}, N^{\prime}}\left(\underline{u}_{(P, \lambda)}\right)_{m, n}= \begin{cases}c_{m, n} & \text { if }|m|<\frac{M}{2} \text { and }|n| \leq \frac{N}{2}, \\ c_{\frac{M}{2}, n}+c_{-\frac{M}{2}, n} & \text { if } m=-\frac{M}{2} \text { and }|n| \leq \frac{N}{2}, \\ 0 & \text { otherwise. }\end{cases}
$$

Note that the case $\lambda_{x}>1$ and $\lambda_{y}=1$ is similarly obtained by switching the role of the dimensions.

3. If $\lambda_{x}>1$ and $\lambda_{y}>1$, then for $(m, n) \in \hat{\Omega}_{M^{\prime}, N^{\prime}}$,

$$
\mathcal{F}_{M^{\prime}, N^{\prime}}\left(\underline{u}_{(P, \lambda)}\right)_{m, n}= \begin{cases}c_{m, n} & \text { if }(m, n) \in \hat{\Omega}_{M, N}^{s}, \\ 0 & \text { otherwise. }\end{cases}
$$

Proof. For $\lambda_{x}=\lambda_{y}=1$ the result is straightforward. Otherwise, for $(k, l) \in \Omega_{M^{\prime}, N^{\prime}}$ we have

$$
\begin{aligned}
\left(\underline{u}_{(P, \lambda)}\right)_{k, l} & =P\left(\frac{k}{\lambda_{x}}, \frac{l}{\lambda_{y}}\right) \\
& =\sum_{(m, n) \in \hat{\Omega}_{M, N}^{s}} c_{m, n} e^{2 i \pi\left(\frac{m k}{\lambda_{x} M}+\frac{n l}{\lambda_{y} N}\right)} \\
& =\sum_{(m, n) \in \hat{\Omega}_{M, N}^{s}} c_{m, n} e^{2 i \pi\left(\frac{m k}{M^{\prime}}+\frac{n l}{N^{\prime}}\right)} .
\end{aligned}
$$


The DFT coefficients are obtained by rewritting the sum over $\hat{\Omega}_{M, N}^{s}$ in (61) as a sum over $\hat{\Omega}_{M^{\prime}, N^{\prime}}$,

$$
\sum_{(m, n) \in \hat{\Omega}_{M, N}^{s}} c_{m, n} e^{2 i \pi\left(\frac{m k}{M^{\prime}}+\frac{n l}{N^{\prime}}\right)}=\sum_{(m, n) \in \hat{\Omega}_{M^{\prime}, N^{\prime}}} d_{m, n} e^{2 i \pi\left(\frac{m k}{M^{\prime}}+\frac{n l}{N^{\prime}}\right)} .
$$

By uniqueness of the iDFT (of size $\left.M^{\prime} \times N^{\prime}\right)$ we have $d=\mathcal{F}_{M^{\prime}, N^{\prime}}\left(\underline{u}_{(P, \lambda)}\right)$. The determination of the coefficients $d$ is done similarly as in the proof of Proposition 1. When a dimension is up-sampled (with a factor strictly larger than 1 ) then the additional coefficients, which are outside of $\hat{\Omega}_{M, N}^{s}$, are set to 0. Otherwise the coefficients are obtained exactly as in Proposition 1.

Therefore the up-sampling using TPI is commonly referred to as the DFT zero-padding. We recall that the trigonometric polynomial coefficients are expressed in terms of the DFT of $\underline{u}$ (see Corollary 1). In particular, for the three classical trigonometric polynomial interpolators the DFT coefficients of the up-sampled images are given by

Proposition 11. Let $\lambda=\left(\lambda_{x}, \lambda_{y}\right) \in\left[1,+\infty\left[{ }^{2}\right.\right.$ verifying $(55)$. For $(m, n) \in \hat{\Omega}_{M^{\prime}, N^{\prime}}$,

$$
\mathcal{F}_{M^{\prime}, N^{\prime}}\left(\underline{u}_{\left(P_{\underline{u}}^{(c)}, \lambda\right)}\right)_{m, n}= \begin{cases}\mathcal{F}_{M, N}(\underline{u})_{m, n} & \text { if }(m, n) \in \hat{\Omega}_{M, N}, \\ 0 & \text { otherwise. }\end{cases}
$$

Assume in addition that $\underline{u}$ is real-valued.

1. If $\lambda_{x}=1$ and $\lambda_{y}>1$, then $M^{\prime}=M$ and for $(m, n) \in \hat{\Omega}_{M^{\prime}, N^{\prime}}$,

$$
\mathcal{F}_{M^{\prime}, N^{\prime}}\left(\underline{u}_{\left(\mathcal{R}\left(P_{\underline{u}}^{(c)}\right), \lambda\right)}\right)_{m, n}=\mathcal{F}_{M^{\prime}, N^{\prime}}\left(\underline{u}_{\left(P_{\underline{u}}^{(r)}, \lambda\right)}\right)_{m, n}= \begin{cases}\mathcal{F}_{M, N}(\underline{u})_{m, n} & \text { if }|n|<\frac{N}{2}, \\ \frac{1}{2} \mathcal{F}_{M, N}(\underline{u})_{m,-\frac{N}{2}} & \text { if }|n|=\frac{N}{2}, \\ 0 & \text { otherwise. }\end{cases}
$$

Note that the case $\lambda_{x}>1$ and $\lambda_{y}=1$ is similarly obtained by switching the role of the dimensions.

2. If $\lambda_{x}>1$ and $\lambda_{y}>1$, then for $(m, n) \in \hat{\Omega}_{M^{\prime}, N^{\prime}}$

$$
\mathcal{F}_{M^{\prime}, N^{\prime}}\left(\underline{u}_{\left(\mathcal{R}\left(P_{\underline{u}}^{(c)}\right), \lambda\right)}\right)_{m, n}= \begin{cases}\mathcal{F}_{M, N}(\underline{u})_{m, n} & \text { if }|m|<\frac{M}{2} \text { and }|n|<\frac{N}{2}, \\ \frac{1}{2} \mathcal{F}_{M, N}(\underline{u})_{-\frac{M}{2}, n} & \text { if }|m|=\frac{M}{2} \text { and }|n|<\frac{N}{2} \\ \frac{1}{2} \mathcal{F}_{M, N}(\underline{u})_{m,-\frac{N}{2}} & \text { if }|m|<\frac{M}{2} \text { and }|n|=\frac{N}{2} \\ \frac{1}{2} \mathcal{F}_{M, N}(\underline{u})_{-\frac{M}{2},-\frac{N}{2}} & \text { if }(m, n) \in\left\{ \pm\left(\frac{M}{2}, \frac{N}{2}\right)\right\} \\ 0 & \text { otherwise. }\end{cases}
$$

and

$$
\mathcal{F}_{M^{\prime}, N^{\prime}}\left(\underline{u}_{\left(P_{\underline{u}}^{(r)}, \lambda\right)}\right)_{m, n}= \begin{cases}\mathcal{F}_{M, N}(\underline{u})_{m, n} & \text { if }|m|<\frac{M}{2} \text { and }|n|<\frac{N}{2}, \\ \frac{1}{2} \mathcal{F}_{M, N}(\underline{u})_{-\frac{M}{2}, n} & \text { if }|m|=\frac{M}{2} \text { and }|n|<\frac{N}{2}, \\ \frac{1}{2} \mathcal{F}_{M, N}(\underline{u})_{m,-\frac{N}{2}} & \text { if }|m|<\frac{M}{2} \text { and }|n|=\frac{N}{2}, \\ \frac{1}{4} \mathcal{F}_{M, N}(\underline{u})_{-\frac{M}{2},-\frac{N}{2}} & \text { if }|m|=\frac{M}{2} \text { and }|n|=\frac{N}{2} \\ 0 & \text { otherwise. }\end{cases}
$$

Proof. It is a direct consequence of Proposition 10. The coefficients of the different trigonometric polynomial interpolators are given in Definition 8, Proposition 2 and Definition 9.

Note that the up-sampled image using the trigonometric polynomial interpolator in complex convention $P_{\underline{u}}^{(c)}$ may be complex-valued for $\underline{u}$ real-valued. Actually it is real-valued if and only if $\left\{(m, n) \in \Gamma_{M, N} \mid \mathcal{F}_{M, N}(\underline{u})_{m, n} \neq 0\right\}=\emptyset$. 
Algorithm. The corresponding algorithm for the up-sampling of an image using TPI is described in Algorithm 3. The total number of floating point operations is in $\mathcal{O}\left(M^{\prime} N^{\prime} \log \left(M^{\prime} N^{\prime}\right)\right)$ as the DFT and iDFT are computed thanks to the FFT algorithm [8].

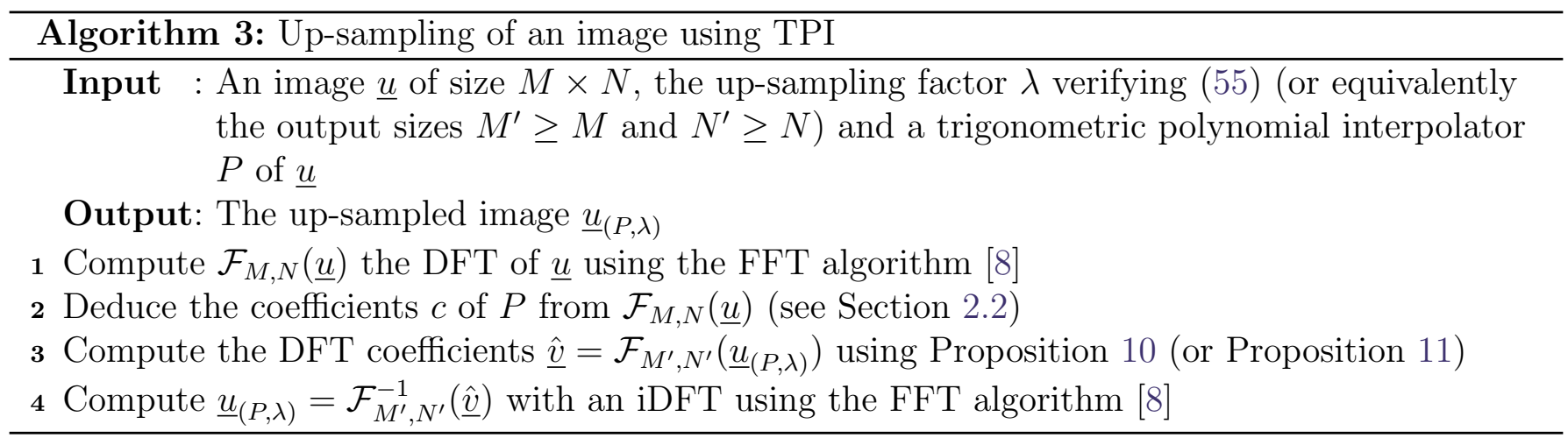

\subsection{Down-sampling}

Here down-sampling refers to the process of reducing the sampling rate of a signal/image. It is also called decimation. For integer down-sampling factors this is usually expressed as a two-step process. First the high-frequency component is reduced by a low-pass filtering. Then, the down-sampled image is obtained by resampling the filtered image on a coarser grid. The aim of the low-pass filtering is to avoid the introduction of a strong aliasing.

Discrete spatial filters may be considered for the low-pass filtering. A classical example is the Gaussian filter (e.g. with standard deviation $\sigma=0.6$ as in $[17,4]$ ).

Low-pass filters may also be defined in the Fourier domain since it covers all the possible image sizes in a single formula and allow for rational down-sampling factors. As described in [5] the filters are then applied using DFT-based computations that rely on TPI. For instance it is the case for the low-pass filter used to build the steerable pyramid of E. Simoncelli et al. [21, 6].

Let $\underline{u}$ be an image of size $M \times N$. Let $\lambda_{x}$ and $\lambda_{y}$ be two numbers greater than 1 such that

$$
\left\{\begin{array}{l}
M^{\prime} \doteq \frac{M}{\lambda_{x}} \in \mathbb{N}, \\
N^{\prime} \doteq \frac{N}{\lambda_{y}} \in \mathbb{N} .
\end{array}\right.
$$

Actually the down-sampling factors $\lambda_{x}=\frac{M}{M^{\prime}}$ and $\lambda_{y}=\frac{N}{N^{\prime}}$ are rational numbers and any downsampled size $M^{\prime} \leq M$ and $N^{\prime} \leq N$ can be obtained. The down-sampling of $\underline{u}$ by the down-sampling factor $\lambda=\left(\lambda_{x}, \lambda_{y}\right)$ using TPI is defined as follows.

Definition 12. Let $M^{\prime}$ and $N^{\prime}$ be two positive integers and $\lambda=\left(\lambda_{x}, \lambda_{y}\right) \in\left[1,+\infty\left[{ }^{2}\right.\right.$. The discrete set $\Lambda_{M^{\prime}, N^{\prime}, \lambda}$ is defined by

$$
\Lambda_{M^{\prime}, N^{\prime}, \lambda}=\Lambda_{M^{\prime}, \lambda_{x}} \times \Lambda_{N^{\prime}, \lambda_{y}}
$$

where for any integer $L$ and $\gamma>1$,

$$
\Lambda_{L, \gamma}= \begin{cases}\hat{\Omega}_{L} & \text { if } \gamma=1, \\ \hat{\Omega}_{L}^{s} & \text { if } \gamma>1 .\end{cases}
$$

Definition 13 (Down-sampling of an image). Let $\lambda=\left(\lambda_{x}, \lambda_{y}\right) \in\left[1,+\infty\left[{ }^{2}\right.\right.$ verifying (67). The down-sampled image of $\underline{u}$ with factor $\lambda$ is noted $\underline{u}_{\left(\frac{1}{\lambda}\right)} \in \mathbb{C}^{\Omega_{M^{\prime}, N^{\prime}}}$. It is defined by

$$
\forall(k, l) \in \Omega_{M^{\prime}, N^{\prime}}, \quad\left(\underline{u}_{\left(\frac{1}{\lambda}\right)}\right)_{k, l}=P_{\underline{u}, \frac{1}{\lambda}}(k, l),
$$


where $P_{\underline{u}, \frac{1}{\lambda}} \in \mathcal{P}_{M^{\prime}, N^{\prime}}$ is given by

$$
\forall(x, y) \in \mathbb{Z}^{2}, \quad P_{\underline{u}, \frac{1}{\lambda}}(x, y)=\sum_{(m, n) \in \Lambda_{M^{\prime}, N^{\prime}, \lambda}} \mathcal{F}_{M, N}(\underline{u})_{m, n} e^{2 i \pi\left(\frac{m}{M^{\prime}} x+\frac{n}{N^{\prime}} y\right)} .
$$

The discrete set $\Lambda_{M^{\prime}, N^{\prime}, \lambda}$ is introduced in Definition 12 in order to handle the cases in Definition 13 where there is no down-sampling along one of the dimensions. To summarize:

- For $\lambda=(1,1)$ there is no effective down-sampling so that we have $\Lambda_{M^{\prime}, N^{\prime}, \lambda}=\hat{\Omega}_{M^{\prime}, N^{\prime}}=\hat{\Omega}_{M, N}$ and $P_{\underline{u}, \frac{1}{\lambda}}=P_{\underline{u}}^{(c)}$.

- When for instance $\lambda_{x}=1$ and $\lambda_{y}>1$, the down-sampling is only performed on the $y$-direction. We have $\Lambda_{M^{\prime}, N^{\prime}, \lambda}=\hat{\Omega}_{M^{\prime}} \times \hat{\Omega}_{N^{\prime}}^{s}=\hat{\Omega}_{M} \times \hat{\Omega}_{N^{\prime}}^{s}$. Note that $\hat{\Omega}_{M}$ is not symmetrical for $M$ even.

- For $\lambda_{x}>1$ and $\lambda_{y}>1$ we have $\Lambda_{M^{\prime}, N^{\prime}, \lambda}=\hat{\Omega}_{M^{\prime}, N^{\prime}}^{s}$, which is a symmetrized discrete Fourier domain.

Contrarily to the up-sampling, the down-sampling of $\underline{u}$ does not depend on a particular choice of an interpolator of $\underline{u}$. The trigonometric polynomial interpolator $P_{\underline{u}}^{(c)}$ is implicitly chosen for the definition of $P_{\underline{u}, \frac{1}{\lambda}}$ but only because it simplifies the notations. Assume that $P \in \mathcal{P}_{M, N}$ is another interpolator of $\underline{u}$ and denote by $c$ its coefficients in $\hat{\Omega}_{M, N}^{s}$. Then, we could replace $P_{\underline{u}, \frac{1}{\lambda}}$ by $\tilde{P}_{\underline{u}, \frac{1}{\lambda}}$ where

$$
\forall(x, y) \in \mathbb{Z}^{2}, \quad \tilde{P}_{\underline{u}, \frac{1}{\lambda}}(x, y)=\sum_{(m, n) \in \hat{\Omega}_{M^{\prime}, N^{\prime}}^{s}} c_{m, n} e^{2 i \pi\left(\frac{m}{M^{\prime}} x+\frac{n}{N^{\prime}} y\right)} .
$$

One can easily prove that

$$
\forall(k, l) \in \Omega_{M^{\prime}, N^{\prime}}, \quad P_{\underline{u}, \frac{1}{\lambda}}(k, l)=\tilde{P}_{\underline{u}, \frac{1}{\lambda}}(k, l),
$$

so that the definition of the down-sampled image $\underline{u}_{\left(\frac{1}{\lambda}\right)}$ would remain unchanged.

Intuitively for $\lambda_{x}>1$ and $\lambda_{y}>1, P_{\underline{u}}, \frac{1}{\lambda}$ is obtained by taking any trigonometric polynomial interpolator of $\underline{u}$, "killing" the coefficients in $\hat{\Omega}_{M, N} \backslash \hat{\Omega}_{M^{\prime}, N^{\prime}}^{s}$ and applying a scaling of factor $\lambda$. The underlying continuous low-pass filter is the perfect low-pass defined by the indicator function of $\left[-\frac{\pi}{\lambda_{x}}, \frac{\pi}{\lambda_{y}}\right]^{2}$.

The down-sampling using TPI is commonly called DFT zoom-out. Indeed, the DFT coefficients of the down-sampled image $\underline{u}_{\left(\frac{1}{\lambda}\right)}$ can be easily computed from DFT coefficients of $\underline{u}$ as stated in

Proposition 12. Let $\lambda=\left(\lambda_{x}, \lambda_{y}\right) \in\left[1,+\infty\left[{ }^{2}\right.\right.$ verifying $(67)$.

1. If $\lambda_{x}=\lambda_{y}=1$, then $\underline{u}_{\left(\frac{1}{\lambda}\right)}=\underline{u}$ and $\mathcal{F}_{M^{\prime}, N^{\prime}}\left(\underline{u}_{\left(\frac{1}{\lambda}\right)}\right)=\mathcal{F}_{M, N}(\underline{u})$.

2. If $\lambda_{x}=1$ and $\lambda_{y}>1$, then $M^{\prime}=M$ and for $(m, n) \in \hat{\Omega}_{M^{\prime}, N^{\prime}}$,

$$
\mathcal{F}_{M^{\prime}, N^{\prime}}\left(\underline{u}_{\left(\frac{1}{\lambda}\right)}\right)_{m, n}= \begin{cases}\mathcal{F}_{M, N}(\underline{u})_{m, n} & \text { if }|n|<\frac{N^{\prime}}{2}, \\ \mathcal{F}_{M, N}(\underline{u})_{m, \frac{N^{\prime}}{2}}+\mathcal{F}_{M, N}(\underline{u})_{m,-\frac{N^{\prime}}{2}} & \text { if } n=-\frac{N^{\prime}}{2} .\end{cases}
$$

Note that the case $\lambda_{x}>1$ and $\lambda_{y}=1$ is similarly obtained by switching the role of the dimensions. 
3. If $\lambda_{x}>1$ and $\lambda_{y}>1$, then for $(m, n) \in \hat{\Omega}_{M^{\prime}, N^{\prime}}$,

$$
\mathcal{F}_{M^{\prime}, N^{\prime}}\left(\underline{u}\left(\frac{1}{\lambda}\right)\right)_{m, n}= \begin{cases}\mathcal{F}_{M, N}(\underline{u})_{m, n} & \text { if }|m|<\frac{M^{\prime}}{2} \text { and }|n|<\frac{N^{\prime}}{2}, \\ \mathcal{F}_{M, N}(\underline{u})_{\frac{M^{\prime}}{2}, n}+\mathcal{F}_{M, N}(\underline{u})_{-\frac{M^{\prime}}{2}, n} & \text { if } m=-\frac{M^{\prime}}{2} \text { and }|n|<\frac{N^{\prime}}{2}, \\ \mathcal{F}_{M, N}(\underline{u})_{m, \frac{N^{\prime}}{2}}+\mathcal{F}_{M, N}(\underline{u})_{m,-\frac{N^{\prime}}{2}} & \text { if }|m|<\frac{M^{\prime}}{2} \text { and } n=-\frac{N^{\prime}}{2}, \\ \mathcal{F}_{M, N}(\underline{u})_{\frac{M^{\prime}}{2}, \frac{N^{\prime}}{2}}+\mathcal{F}_{M, N}(\underline{u})_{\frac{M^{\prime}}{2},-\frac{N^{\prime}}{2}} & \\ +\mathcal{F}_{M, N}(\underline{u})_{-\frac{M^{\prime}}{2}, \frac{N^{\prime}}{2}}+\mathcal{F}_{M, N}(\underline{u})_{-\frac{M^{\prime}}{2},-\frac{N^{\prime}}{2}} & \text { if } m=-\frac{M^{\prime}}{2} \text { and } n=-\frac{N^{\prime}}{2} .\end{cases}
$$

Proof. It is obtained by using similar computations as in the proof of Proposition 1.

Assume that $\underline{u}$ is real-valued. Then, using the symmetry of $\mathcal{F}_{M, N}(\underline{u})$ one can easily prove that the down-sampled image $\underline{u}_{\left(\frac{1}{\lambda}\right)}$ is also real-valued.

Algorithm. The corresponding algorithm for the down-sampling of an image using TPI is described in Algorithm 4. The total number of floating point operations is in $\mathcal{O}(M N \log (M N))$ as the DFT and iDFT are computed thanks to the FFT algorithm [8].

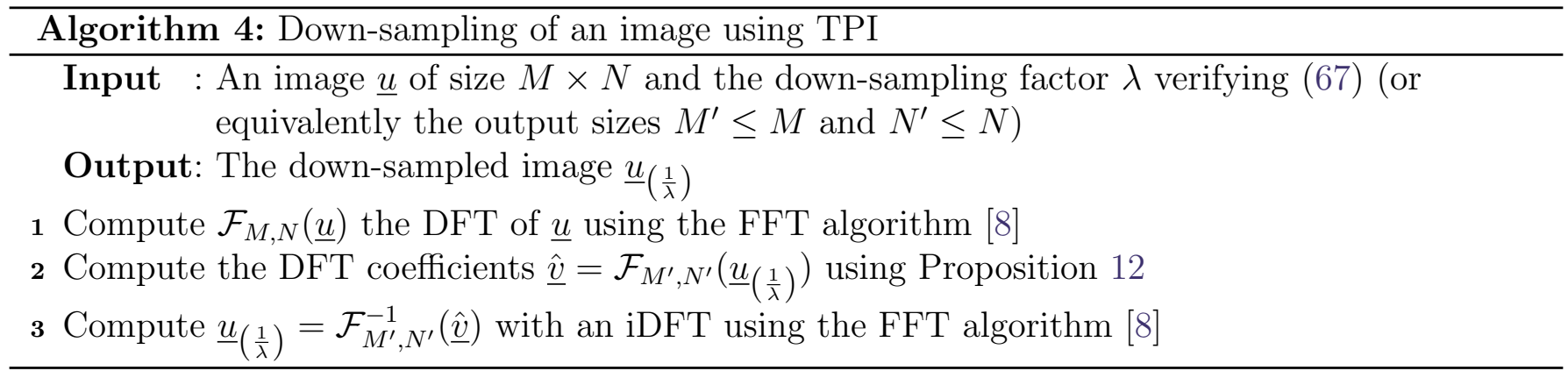

\subsection{Link between Up-sampling and Down-sampling}

The left invertibility of the up-sampling using TPI is guaranteed by

Proposition 13. Let $\lambda=\left(\lambda_{x}, \lambda_{y}\right) \in\left[1,+\infty\left[^{2}\right.\right.$ verifying (55). Let $P \in \mathcal{P}_{M, N}$ be a trigonometric polynomial interpolator of $\underline{u}$. Then,

$$
\left(\underline{u}_{(P, \lambda)}\right)_{\left(\frac{1}{\lambda}\right)}=\underline{u} .
$$

Proof. It is obtained using Proposition 10 and Proposition 12.

More precisely, the left inverse of the up-sampling with any trigonometric polynomial interpolator is the down-sampling with the same factor. Obviously for $\lambda \neq(1,1)$ the up-sampling does not admit a right inverse since up-sampled images have imposed null DFT coefficients. Similarly the downsampling does not admit a left inverse since DFT coefficients are "killed" i.e. some information is lost. 


\section{Provided Implementation and Online Demo}

We propose ANSI C99 implementations of the DFT translation (Algorithm 1), of the geometric transformation (Algorithm 2) by an homography, of the up-sampling (Algorithm 3) and of the downsampling (Algorithm 4). The source codes and an online demo are available at the IPOL web page of this article ${ }^{2}$.

Our implementations handle the two classical real-valued interpolators but not the complex one as only real-valued images can commonly be saved. For the FFT and NFFT algorithms we use the FFTW library [10] and the NFFT3 library [12].

\section{$6 \quad$ Experiments}

In this experimental section we present some results obtained using the described algorithms (and the corresponding proposed implementations). The input image of size $584 \times 388$ that we use is shown in Figure 2. This is the grayscale version of the RubberWhale image from the Middlebury database [2].

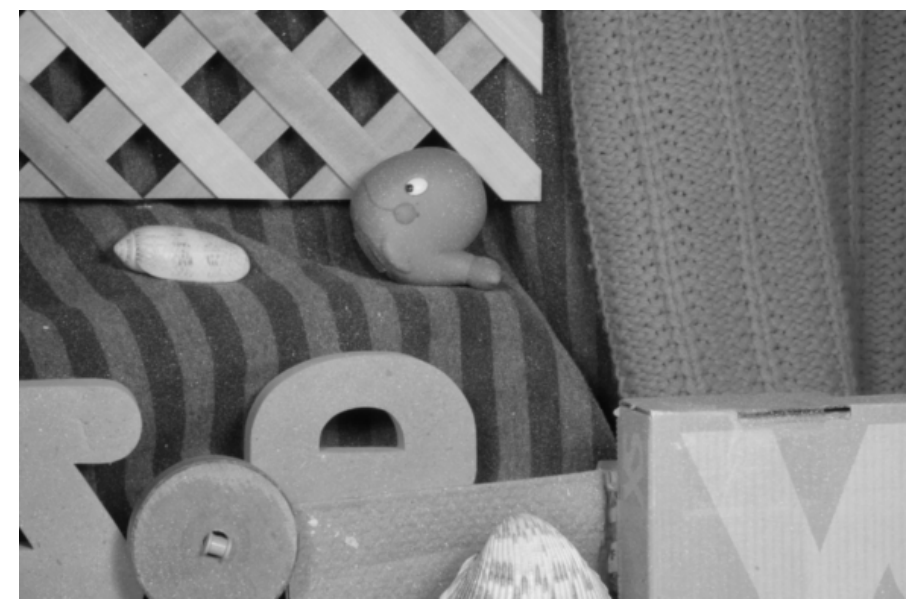

Figure 2: Input image of size $584 \times 388$ used for the experiments in Section 6 . This is the grayscale version of the RubberWhale image from the Middlebury database [2].

In all of the presented experiments we used the trigonometric polynomial interpolator in real convention. A study of the impact of the choice of the trigonometric polynomial interpolator was made in [5] in a context of filtering. The experiments were made using an Intel(R) Core(TM) i7-7820HQ CPU.

\subsection{DFT translation}

We applied the shift $(100.5,100.5)$ using the DFT translation algorithm described in Algorithm 1. The shifted image, shown in Figure 3(a), was obtained in 40ms. Typical ringing artifacts, occurring around the image boundary because of the inadequate periodic assumption, can be seen in Figure 3(b).

According to Proposition 9, the translation by $(100.5,100.5)$ of our input image is not invertible. To verify this, we applied the opposite shift $-(100.5,100.5)$ to the shifted image and compared the result to the original image. The maximal error in absolute value is of 0.70 and the root mean square error (RMSE) is of 0.18. Depending on the context of application, this error, which is localized in the Fourier boundary $\Gamma_{M, N}$, may be non-negligible.

\footnotetext{
${ }^{2}$ https://doi.org/10.5201/ipol.2019.273
} 


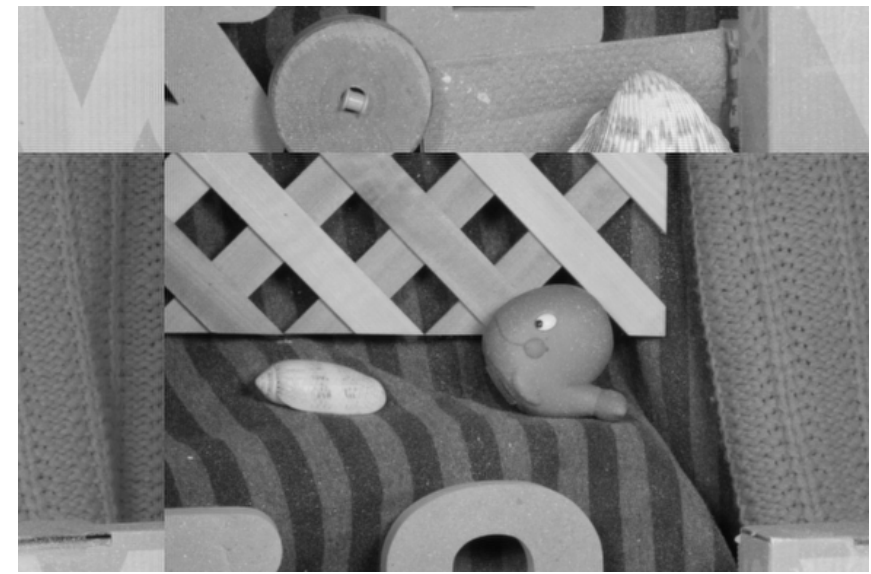

(a) Shifted image by $(100.5,100.5)$

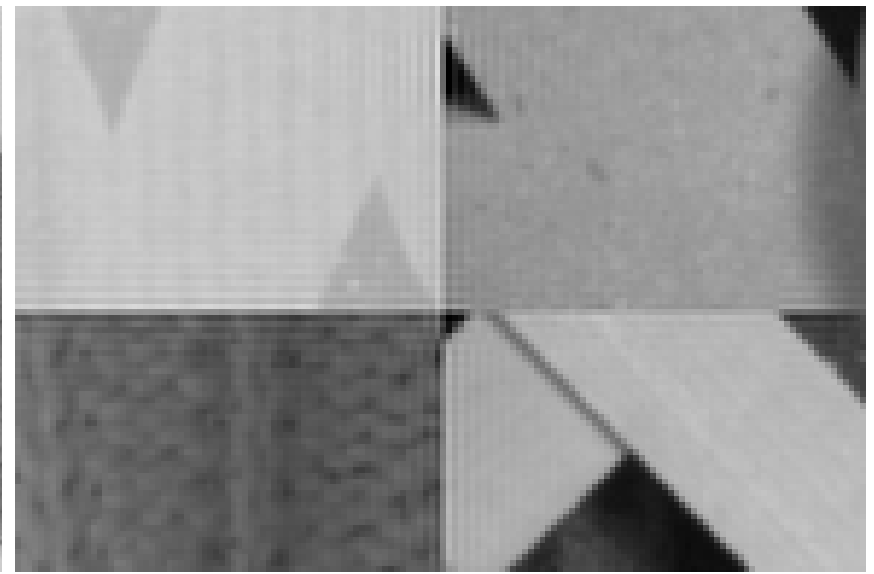

(b) Detail of (a) around $(100,100)$

Figure 3: Results of the DFT translation of the image presented in Figure 2 for the shift $(100.5,100.5)$. The shifted image, shown in (a), was obtained in $40 \mathrm{~ms}$. Typical ringing artifacts, occurring around the image boundary because of the inadequate periodic assumption, can be seen in (b).

\subsection{Geometric Transformation}

In order to compare the geometric transformation algorithm, which uses the NFFT algorithm, and the DFT translation algorithm, we also applied the shift of $(100.5,100.5)$ using Algorithm 2. The maximal difference between the two results was around $10^{-10}$ in absolute value. Indeed, the NFFT algorithm precision is close to the machine precision. However for translations, the DFT computations are way more efficient as the NFFT version took 1850ms (against 40ms).

The advantage of Algorithm 2 is to allow for any geometric transformation. As an example, we used it for the rotation of $45^{\circ}$ centered in $(0,0)$ (i.e. the top-left corner of the image). The transformed image is shown in Figure 4.

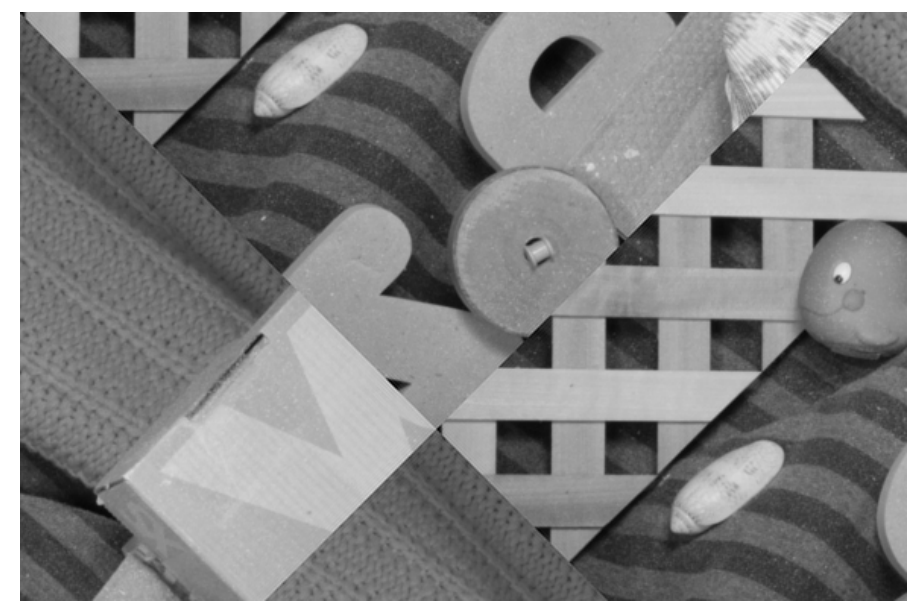

Figure 4: Results of the geometric transformation of the image presented in Figure 2 for the rotation of $45^{\circ}$ centered in $(0,0)$ (i.e. the top-left corner of the image).

\subsection{Up-sampling}

We applied the up-sampling of factors $(2,2),(2.5,2.5)$ and $(3,3)$ using the up-sampling algorithm described in Algorithm 3. The results are presented in Figure 5. 
Note that because of the inadequate periodic assumption ringing may appear after up-sampling. In [1] the authors proposed to first use the periodic plus smooth decomposition [14]. Then, the periodic component is up-sampled using the DFT up-sampling method while the smooth component is up-sampled using another interpolation method (e.g. using bilinear interpolation).

\subsection{Down-sampling}

We applied the down-sampling of factors $\left(\frac{584}{400}, \frac{388}{200}\right),(2,2)$ and $(4,4)$ using the down-sampling algorithm described in Algorithm 4. The first factor was chosen in order to get an output image of size $400 \times 200$. The results are presented in Figure 6 .

\section{Conclusion}

In this work we presented a trigonometric polynomial interpolation theory for images and applied it to the geometric transformation of images, to the up-sampling and to the down-sampling. The trigonometric polynomial interpolators of an image were characterized and it was shown that there are infinitely many candidates as soon as one of the image dimensions is even. The interpolator choice has an influence as shown in the two discussed applications. Three classical choices of interpolator for real-valued images were presented and the cases where they coincide were pointed out.

For image translation, the classical DFT-based computations by phase shift were described. In the general case an efficient but approximate algorithm, based on the NFFT algorithm, was proposed. DFT-based computations were also presented for the up-sampling and the down-sampling. All of the algorithms described are efficient and can be used in practice. Trigonometric polynomial interpolation can also be applied to linear filtering as in [5].

The performances, the limits and the improvements of trigonometric polynomial interpolation are discussed in [3, Chapter 6].

\section{Acknowledgements}

The authors would like to thank Prof. Jean-Michel Morel for his support, suggestions, and many fruitful discussions.

Work partly financed by Office of Naval research grant N00014-17-1-2552, DGA Astrid project "filmer la Terre" no ANR-17-ASTR-0013-01, MENRT and Fondation Mathématique Jacques Hadamard.

\section{Image Credits}

Grayscale version of the Rubber Whale image from the Middlebury database [2] 


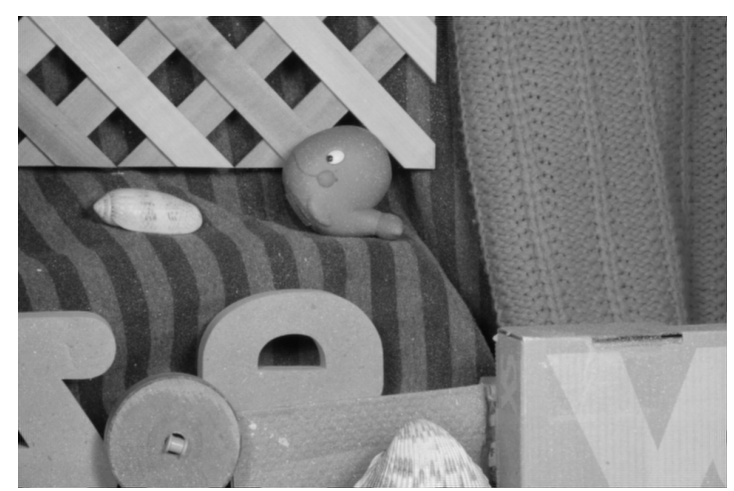

(a) Up-sampling by factor $(2,2)$. Done in $50 \mathrm{~ms}$. The output size is $1168 \times 776$.

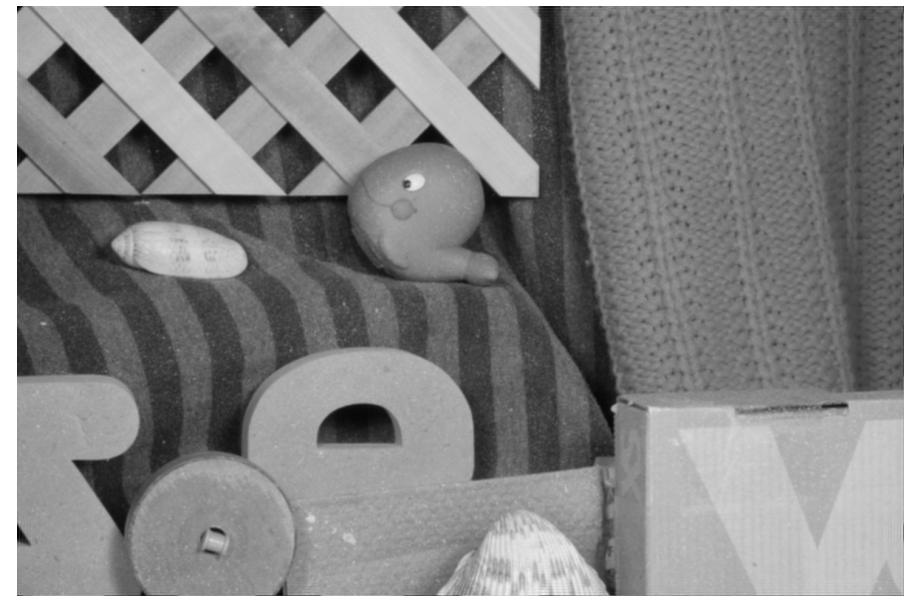

(b) Up-sampling by factor $(2.5,2.5)$. Done in $70 \mathrm{~ms}$. The output size is $1460 \times 960$.

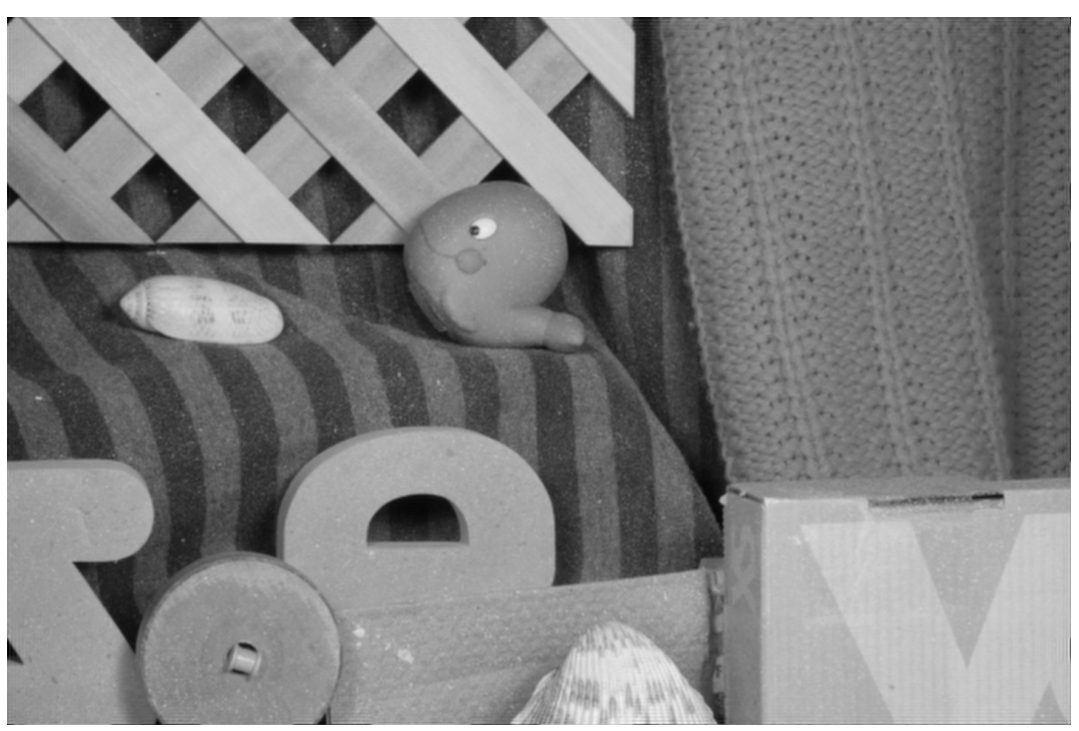

(c) Up-sampling by factor $(3,3)$. Done in $95 \mathrm{~ms}$. The output size is $1752 \times 1164$

Figure 5: Results of the up-sampling of the image presented in Figure 2 for various up-sampling factors. The images are displayed at dimensions proportional to their zoom factor. 


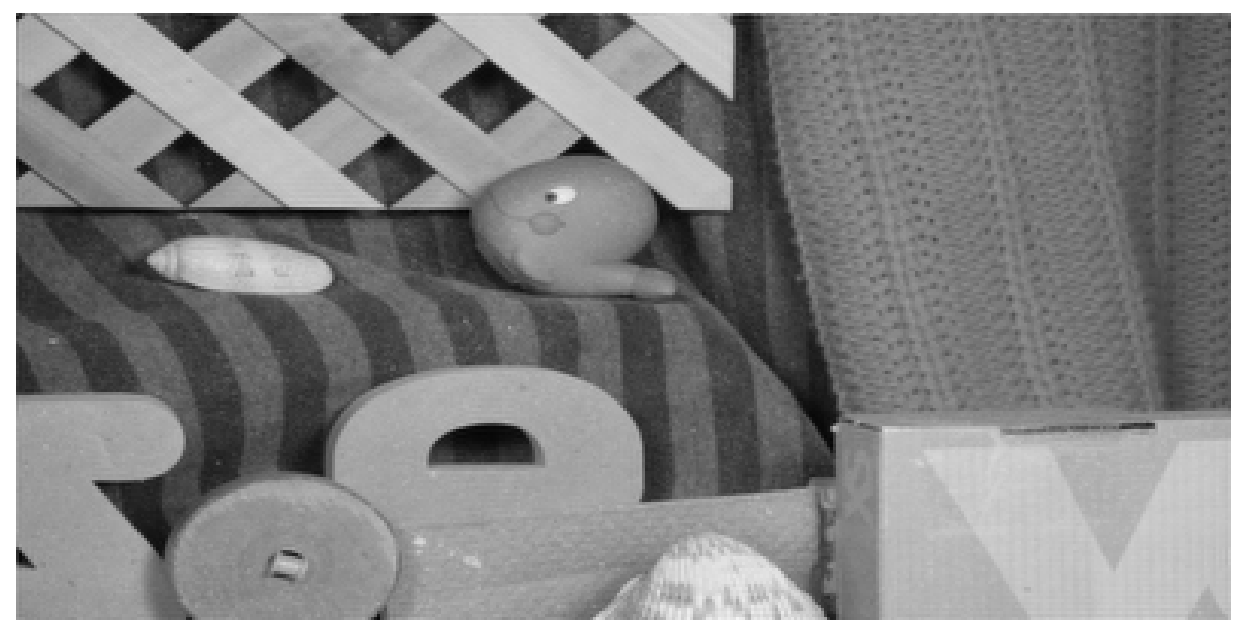

(a) Down-sampling by factor $\left(\frac{584}{400}, \frac{388}{200}\right)$. Done in $17 \mathrm{~ms}$. The output size is $400 \times$ 200 .

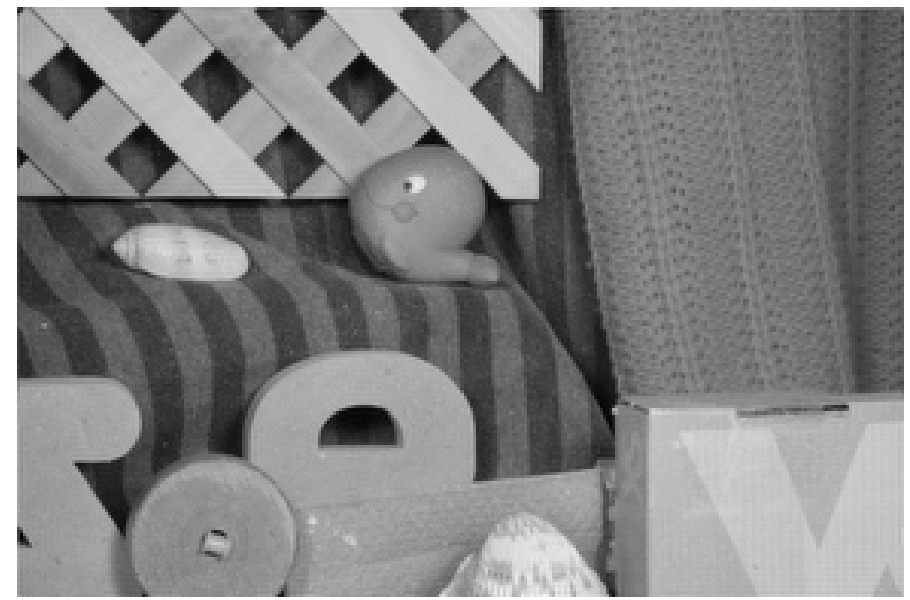

(b) Down-sampling by factor $(2,2)$. Done in $20 \mathrm{~ms}$. The output size is $292 \times 194$.

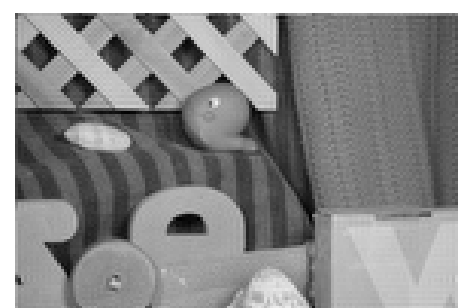

(c) Down-sampling by factor $(4,4)$. Done in $28 \mathrm{~ms}$. The output size is $146 \times 97$.

Figure 6: Results of the down-sampling of the image presented in Figure 2 for various down-sampling factors. The images are displayed at dimensions proportional to their zoom factor. Note that the computation time does not necessarily decrease as the output size decreases because of optimizations made in the FFTW library [10]. 


\section{References}

[1] R. Abergel and L. Moisan, The Shannon Total Variation, Journal of Mathematical Imaging and Vision, (2017), pp. 1-30. http://dx.doi.org/10.1007/s10851-017-0733-5.

[2] S. Baker, D. Scharstein, J. P. Lewis, S. Roth, M. J. Black, and R. Szeliski, A Database and Evaluation Methodology for Optical Flow, International Journal of Computer Vision, 92 (2011), pp. 1-31. http://dx.doi.org/10.1007/s11263-010-0390-2.

[3] T. BRIAnd, Image Formation from a Large Sequence of RAW Images : performance and accuracy, PhD Thesis, Université Paris-Est, Nov. 2018. https://pastel.archives-ouvertes.fr/ tel-01980492/file/TH2018PESC1017.pdf.

[4] T. Briand, G. Facciolo, And J. SÁnchez, Improvements of the Inverse Compositional Algorithm for Parametric Motion Estimation, Image Processing On Line, 8 (2018), pp. 435464. http://dx.doi.org/10.5201/ipol.2018.222.

[5] T. Briand and J. Vacher, How to Apply a Filter Defined in the Frequency Domain by a Continuous Function, Image Processing On Line, 6 (2016), pp. 183-211. http://dx.doi.org/ 10.5201/ipol.2016.116.

[6] T. Briand, J. Vacher, B. Galerne, and J. Rabin, The Heeger $\&$ Bergen Pyramid Based Texture Synthesis Algorithm, Image Processing On Line, 4 (2014), pp. 276-299. http://dx. doi.org/10.5201/ipol.2014.79.

[7] E.W. Cheney, Approximation theory III, vol. 12, Academic Press New York, 1980. ISBN 9780121710507.

[8] J. W CoOley And J. Tukey, An algorithm for the machine calculation of complex Fourier series, Mathematics of Computation, 19 (1965), pp. 297-301. http://dx.doi.org/10.2307/ 2003354.

[9] P. Dienckx, Curve and surface fitting with splines, Oxford University Press, 1995 . ISBN 9780198534402.

[10] M. Frigo And S.G. Johnson, The Design and Implementation of FFTW3, Proceedings of the IEEE, 93 (2005), pp. 216-231. http://dx.doi.org/10.1109/JPROC.2004.840301.

[11] Pascal Getreuer, Linear Methods for Image Interpolation, Image Processing On Line, 1 (2011), pp. 238-259. https://doi.org/10.5201/ipol.2011.g_lmii.

[12] J. KeIner, S. Kunis, And D. PotTs, Using NFFT 3-a software library for various nonequispaced fast Fourier transforms, ACM Transactions on Mathematical Software (TOMS), 36 (2009), p. 19. http://dx.doi.org/10.1145/1555386.1555388.

[13] S. Kunis And D. PotTs, Time and memory requirements of the Nonequispaced FFT, Sampling Theory in Signal and Image Processing, 7 (2008), pp. 77-100.

[14] L. Moisan, Periodic plus smooth image decomposition, Journal of Mathematical Imaging and Vision, 39 (2011), pp. 161-179. http://dx.doi.org/10.1007/s10851-010-0227-1.

[15] D. Potts, G. Steidl, And M. Tasche, Fast Fourier Transforms for Nonequispaced Data: A Tutorial, Birkhäuser Boston, Boston, MA, 2001, pp. 247-270. http://dx.doi.org/10.1007/ 978-1-4612-0143-4_12. 
[16] W. Rudin, Real and complex analysis, Tata McGraw-Hill Education, 1987. ISBN 0070542341.

[17] J. SÁnchez, The Inverse Compositional Algorithm for Parametric Registration, Image Processing On Line, 6 (2016), pp. 212-232. http://dx.doi.org/10.5201/ipol.2016.153.

[18] L. Schwartz, Théorie des distributions, Actualités Scientifiques et Industrielles, Institut de Mathématique, Université de Strasbourg, 1 (1966), p. 2. http://dx.doi.org/10.1090/ S0002-9904-1952-09555-0.

[19] C.E. Shannon, Communication in the Presence of Noise, Proceedings of the IRE, 37 (1949), pp. 10-21. http://dx.doi.org/10.1109/JRPROC.1949.232969.

[20] L. Simon And J-M. Morel, Influence of Unknown Exterior Samples on Interpolated Values for Band-limited Images, SIAM Journal on Imaging Sciences, 9 (2016), pp. 152-184. http: //dx.doi.org/10.1137/140978338.

[21] E.P. Simoncelli And W.T. Freeman, The steerable pyramid: A flexible architecture for multi-scale derivative computation, in Proceedings of the International Conference on Image Processing, vol. 3, IEEE, 1995, pp. 444-447. http://dx.doi.org/10.1109/ICIP.1995.537667.

[22] R.S. Strichartz, A guide to distribution theory and Fourier transforms, World Scientific, 2003. ISBN 0849382734.

[23] P. Thévenaz, T. Blu, And M. Unser, Interpolation Revisited [Medical Images Application], IEEE Transactions on Medical Imaging, 19 (2000), pp. 739-758. http://dx.doi.org/10.1109/ 42.875199 .

[24] E. T. WhitTaker, XVIII. On the functions which are represented by the expansions of the interpolation theory, Proceedings of the Royal Society of Edinburgh, 35 (1915), pp. 181-194. http://dx.doi.org/10.1017/S0370164600017806. 